\title{
Long-time behaviour of a non-autonomous parabolic equation with nonlocal diffusion and sublinear terms
}

\author{
Tomás Caraballo, Marta Herrera-Cobos, and Pedro Marín-Rubio \\ Dpto. Ecuaciones Diferenciales y Análisis Numérico \\ Universidad de Sevilla \\ Apdo. de Correos 1160, 41080-Sevilla, Spain \\ E-mail addresses: caraball@us.es, mhc@us.es,pmr@us.es \\ Dedicated to Enzo Mitidieri on occasion of his Sixtieth Birthday
}

\begin{abstract}
This paper is devoted to study the asymptotic behaviour of a time-dependent parabolic equation with nonlocal diffusion and nonlinear terms with sublinear growth. Namely, we extend some previous results from the literature, obtaining existence, uniqueness, and continuity results, analyzing the stationary problem and decay of the solutions of the evolutionary problem, and finally, under more general assumptions, ensuring the existence of pullback attractors for the associated dynamical system in both $L^{2}$ and $H^{1}$ norms. Relationship among these objects are established using regularizing properties of the equation.
\end{abstract}

2010 MSC: 35B40, 35B41, 35B65, 35K55, 35Q92, 37L30.

Key words and phrases: nonlocal diffusion, nonlocal terms, pullback attractors, regularity of attractors.

\section{Introduction}

In recent decades, nonlocal problems have arisen in modeling with great interest by its usefulness in real applications (e.g. cf. [18, 4, 20, 30,3]). For instance, in Biology, the evolution of some species might be better represented by a nonlocal equation than within the corresponding local simplification. Namely, as example of a spatial nonlocal feature, the migration of population of bacterias in a container depends on the global population in a certain subdomain (e.g. cf. $[10,11])$. Of course, the disadvantage is that sometimes it is very complicated to deal with the nonlocal operators and terms since they are more involved.

In particular, much attention has been paid to the parabolic PDE with nonlocal diffusion

$$
\frac{d u}{d t}-a(l(u)) \Delta u=f
$$

where the function $a$ appearing in the diffusion coefficient satisfies suitable assumptions (detailed below) and depends on a nonlocal functional applied to the solution $u$. For instance, see [24, 9] for a detailed introduction on different applications, and for a very refined analysis of why imposing natural conditions of non-degeneracy of $a$ (controlled from above and below) in order to avoid extinction and only existence of solutions in finite-time intervals.

This problem is far away of being a trivial perturbation of a heat equation, since the nonlinear diffusion makes that the Lyapunov structure is, in general, lost, and manipulations that can be done in the local case are not valid here (e.g. roughly speaking, one cannot gain some information on a priori estimates by multiplying by $u_{t}$ ). 
Despite the above obstacles, the qualitative analysis of the evolution of solutions to the problem can be related to interesting questions as periodicity, or asymptotic-in-time issues -permanence vs extinction phenomena, stability-, etcetera, with meaningful interpretations, but more involved technical difficulties, which can only be solved in special cases.

To be more precise, the results by Chipot and his collaborators (cited below) are referred to the above equation with $f$ independent of $u$. There, besides existence and uniqueness results by different ways, the evolution for long times of the solutions is established under additional assumptions and particular suitably ordered initial conditions w.r.t. stationary solutions (e.g. see [10, 12]) or [13] for a mixed formulation in the boundary. As said before, a kind of Lyapunov structure were available only in very special situations (see $[12,14,15]$ ). Another modification of the above equation, with the nonlocal term in another position rather than in the diffusion, was also treated in [8], with similar conclusions (see also [7] for a model that includes as particular cases those cited above).

In $[1,2]$ the authors analyze the case in which $f$ is still independent of $u$, and the nonlocal operator is not acting globally in the whole domain but in the part of it contained in a ball centered on each position point. Radial solutions, bifurcation analysis, branch of solutions and their stability are studied.

For $f$ depending on the unknown $u$ in a semilinear form, the situation is more involved. In [29], by fixed point techniques, it is proved the existence and uniqueness of weak solution of a semilinear problem with nonlocal diffusion where the domain $\Omega$ has smooth boundary, and the term $f$ (depending on $u$ ) is a Lipschitz continuous function. In addition, the existence and uniqueness of periodic solutions is also analyzed.

Concerning the long-time behaviour of solutions, it is also worth considering the information that can be obtained by the theory of attractors, under less restrictive conditions on the problem. When $f$ is independent of the solution and of time, this was also addressed by Andami [1] (see also some previous results on this direction by Lovat [24]).

However, in this context of attractors, there are more choices to deal with, rather than the compact global attractor for an autonomous dynamical system. Indeed, after including time-dependent terms, which allows to model more complex situations, there are several different approaches from the point of view of non-autonomous dynamical systems, as uniform attractors, skew-product flows, and pullback attractors (e.g. cf. [22]; also related to random dynamical systems, cf. [17]). All of them are valid to analyze different features of the evolution of a non-autonomous dynamical system. We choose that of pullback attractors since it allows us to minimize the assumptions on the forcing terms, and the resultant objects are strictly invariant (in a suitable "non-autonomous-dynamical-system sense"). In this last approach of pullback attractors, many new results have appeared over the last years, allowing, as in the random case, to deal with not only fixed bounded sets, but developing the concept of attraction of a class of families (specifically, a universe $\mathcal{D}$ ) parameterized in time, which usually appears in applications and use to be defined in term of a tempered condition (e.g. cf. [16, 5, 6]). Relationships between both type of attractors have already been established, and under suitable assumptions, they may actually coincide (cf. [27]).

The goal of this paper is to study the existence and regularity of pullback attractors for dynamical systems generated by solutions of a parabolic PDE as above but of semilinear type, with nonlocal diffusion, and additional non-autonomous terms, extending the previously cited results. As far as we know, in the previous literature there is no study on the existence of the pullback attractor in $L^{2}(\Omega)$ nor $H_{0}^{1}(\Omega)$ for the associated dynamical system, as we will consider in this paper. As a first step towards this general aim, here we will be concerned with the sublinear case, to avoid additional difficulties that appear and which will be analyzed elsewhere in future.

The content of the paper is as follows. In Section 2 we state the problem and some definitions of solutions and energy equalities that will be used later, and establish some existence results by compactness arguments. Section 3 is devoted to analyze the existence of stationary solution (combining 
fixed point and compactness arguments), and uniqueness, under additional assumptions. We conclude this paragraph studying the global exponential stability of this steady state, i.e. exponential decay of the solutions to the evolution problem toward this unique stationary solution. In Section 4 we briefly recall some abstract results on pullback attractors that will be used in the sequel. Then, in Section 5 we establish the existence of several pullback attractors in $L^{2}(\Omega)$. The proof is based on an energy method which relies on the continuity of solutions. In addition, we establish some relationships between these pullback attractors. Finally, in Section 6 we use a similar argument to ensure the existence of pullback attractors in $H_{0}^{1}(\Omega)$. Moreover, under suitable assumptions and taking into account the regularizing effect of the equation, we can establish relationships between these families of pullback attractors.

\section{Statement of the problem and existence results}

Consider the following problem for a nonlocal and nonlinear parabolic equation:

$$
\left\{\begin{array}{l}
\frac{d u}{d t}-a(l(u)) \Delta u=f(u)+h(t) \quad \text { in } \Omega \times(\tau,+\infty) \\
u=0 \text { on } \partial \Omega \times(\tau,+\infty) \\
u(x, \tau)=u_{\tau}(x) \text { in } \Omega
\end{array}\right.
$$

where $\Omega \subset \mathbb{R}^{N}$ is a bounded open set, $\tau \in \mathbb{R}, a \in C\left(\mathbb{R} ; \mathbb{R}_{+}\right)$is locally Lipschitz, and there exist positive constants $m, M$ such that

$$
0<m \leq a(s) \leq M \quad \forall s \in \mathbb{R}
$$

$l \in\left(L^{2}(\Omega)\right)^{\prime}, f \in C(\mathbb{R})$ and there exist constants $\eta>0$, and $C_{f} \geq 0$, such that

$$
\begin{aligned}
|f(s)| & \leq C_{f}(1+|s|) \quad \forall s \in \mathbb{R} \\
(f(s)-f(r))(s-r) & \leq \eta(s-r)^{2} \quad \forall s, r \in \mathbb{R} .
\end{aligned}
$$

As usual, we will denote by $(\cdot, \cdot)$ the inner product in $L^{2}(\Omega)$ and by $|\cdot|$ its associated norm (since no confusion arises, this will also denote the Lebesgue measure of a subset of $\left.\mathbb{R}^{N}\right)$. By $((\cdot, \cdot))$ we will denote the inner product in $H_{0}^{1}(\Omega)$ given by the product in $\left(L^{2}(\Omega)\right)^{N}$ of the gradients, by $\|\cdot\|$ the associated norm, by $\langle\cdot, \cdot\rangle$ the duality product between $H^{-1}(\Omega)$ and $H_{0}^{1}(\Omega)$, and by $\|\cdot\|_{*}$ the norm in $H^{-1}(\Omega)$. Identifying $L^{2}(\Omega)$ with its dual, we have the usual chain of dense and compact embeddings $H_{0}^{1}(\Omega) \subset L^{2}(\Omega) \subset H_{\tilde{l}}^{-1}(\Omega)$. Observe that, by the Riesz theorem, we can obtain $\tilde{l} \in L^{2}(\Omega)$ with $\langle l, u\rangle_{\left(L^{2}(\Omega)\right)^{\prime}, L^{2}(\Omega)}=(\tilde{l}, u)$; here on, thanks to the identification $\left(L^{2}(\Omega)\right)^{\prime} \equiv L^{2}(\Omega)$, we will make the abuse of notation of using $l$ instead of $\tilde{l}$, but at the same time we keep the usual notation in the existing previous literature $l(u)$ instead of $(l, u)$ for the operator $l$ acting on $u$.

In what follows, we assume that $u_{\tau} \in L^{2}(\Omega)$ and $h \in L_{\text {loc }}^{2}\left(\mathbb{R} ; H^{-1}(\Omega)\right)$.

Definition 1. A weak solution to (1) is a function $u$ that belongs to $L^{2}\left(\tau, T ; H_{0}^{1}(\Omega)\right) \cap L^{\infty}\left(\tau, T ; L^{2}(\Omega)\right)$ for all $T>\tau$, with $u(\tau)=u_{\tau}$, such that

$$
\frac{d}{d t}(u(t), v)+a(l(u(t)))((u(t), v))=(f(u(t)), v)+\langle h(t), v\rangle \quad \forall v \in H_{0}^{1}(\Omega),
$$

where the previous equation must be understood in the sense of $\mathcal{D}^{\prime}(\tau,+\infty)$.

Remark 2. If $u$ is a weak solution to (1), then (2), (3), and (5) imply that $u^{\prime} \in L^{2}\left(\tau, T ; H^{-1}(\Omega)\right)$ for any $T>\tau$, and therefore $u \in C\left([\tau,+\infty) ; L^{2}(\Omega)\right)$. Hence the initial datum in (1) makes sense. Moreover, we have the following energy equality for all $\tau \leq s \leq t$ :

$$
|u(t)|^{2}+2 \int_{s}^{t} a(l(u(r)))\|u(r)\|^{2} d r=|u(s)|^{2}+2 \int_{s}^{t}(f(u(r)), u(r)) d r+2 \int_{s}^{t}\langle h(r), u(r)\rangle d r .
$$


A notion of more regular solution is also suitable for the problem.

Definition 3. A strong solution to (1) is a weak solution $u$ which also satisfies that $u \in L^{2}(\tau, T ; D(-\Delta))$ $\cap L^{\infty}\left(\tau, T ; H_{0}^{1}(\Omega)\right)$ for all $T>\tau$.

Remark 4. If $h \in L_{\text {loc }}^{2}\left(\mathbb{R} ; L^{2}(\Omega)\right)$ and $u$ is a strong solution to (1), then $u^{\prime} \in L^{2}\left(\tau, T ; L^{2}(\Omega)\right)$ for all $T>\tau$, and, consequently, $u \in C\left([\tau,+\infty) ; H_{0}^{1}(\Omega)\right)$. In addition, the following energy equality holds:

$$
\|u(t)\|^{2}+2 \int_{s}^{t} a(l(u(r)))|-\Delta u(r)|^{2} d r=\|u(s)\|^{2}+2 \int_{s}^{t}(f(u(r))+h(r),-\Delta u(r)) d r \forall \tau \leq s \leq t .
$$

Next result establishes the existence and uniqueness of weak and strong solution to (1), the regularizing effect of the equation, and the continuity of the solution in $L^{2}(\Omega)$ with respect to initial data.

Theorem 5. Assume that the function a is locally Lipschitz and satisfies (2), $f \in C(\mathbb{R})$ fulfills (3) and (4), and consider $h \in L_{\text {loc }}^{2}\left(\mathbb{R} ; H^{-1}(\Omega)\right)$ and $l \in L^{2}(\Omega)$ given. Then, for each $u_{\tau} \in L^{2}(\Omega)$, the problem (1) possesses a unique weak solution, denoted by $u(\cdot)=u\left(\cdot ; \tau, u_{\tau}\right)$. Moreover, this solution behaves continuously in $L^{2}(\Omega)$ w.r.t. initial data.

In addition, if $h \in L_{l o c}^{2}\left(\mathbb{R} ; L^{2}(\Omega)\right)$, for every $\varepsilon>0$ and $T>\tau+\varepsilon$, this solution u satisfies that $u \in C\left((\tau, T], H_{0}^{1}(\Omega)\right) \cap L^{2}(\tau+\varepsilon, T ; D(-\Delta))$. In fact, if the initial condition $u_{\tau} \in H_{0}^{1}(\Omega)$, then $u \in C\left([\tau, T], H_{0}^{1}(\Omega)\right) \cap L^{2}(\tau, T ; D(-\Delta))$ for every $T>\tau$, i.e. $u$ is a strong solution.

Proof. We split the proof into three steps.

Step 1. Uniqueness of solution and continuity w.r.t. initial data. We will prove both assertions simultaneously since the same estimates are valid for both purposes. Suppose that $u_{1}$ and $u_{2}$ are two weak solutions to (1) corresponding to initial values $u_{1 \tau}, u_{2 \tau} \in L^{2}(\Omega)$ respectively. From the energy equality,

$$
\begin{aligned}
& \frac{1}{2} \frac{d}{d t}\left|u_{1}(t)-u_{2}(t)\right|^{2}+a\left(l\left(u_{1}(t)\right)\right)\left\|u_{1}(t)-u_{2}(t)\right\|^{2} \\
= & {\left[a\left(l\left(u_{2}(t)\right)\right)-a\left(l\left(u_{1}(t)\right)\right)\right]\left(\left(u_{2}(t), u_{1}(t)-u_{2}(t)\right)\right)+\left(f\left(u_{1}(t)\right)-f\left(u_{2}(t)\right), u_{1}(t)-u_{2}(t)\right) }
\end{aligned}
$$

a.e. $t \in[\tau, T]$.

Since $u_{1}, u_{2} \in C\left([\tau, T] ; L^{2}(\Omega)\right)$, it holds that $u_{1}(t), u_{2}(t) \in S$ for all $t \in[\tau, T]$, where $S$ is a bounded set of $L^{2}(\Omega)$. On the other hand, as $l \in L^{2}(\Omega)$, there exists a value $R>0$ such that $\left\{l\left(u_{i}(t)\right)\right\}_{t \in[\tau, T]} \subset[-R, R]$ for $i=1,2$. Hence, using (2), (4), and the locally Lipschitz continuity of the function $a$, it yields

$$
\begin{aligned}
& \frac{1}{2} \frac{d}{d t}\left|u_{1}(t)-u_{2}(t)\right|^{2}+m\left\|u_{1}(t)-u_{2}(t)\right\|^{2} \\
\leq & L_{a}(R)|l|\left|u_{1}(t)-u_{2}(t)\right|\left\|u_{2}(t)\right\|\left\|u_{1}(t)-u_{2}(t)\right\|+\eta\left|u_{1}(t)-u_{2}(t)\right|^{2},
\end{aligned}
$$

where $L_{a}(R)$ is the Lipschitz constant of the function $a$ in $[-R, R]$. Thanks to the Young inequality,

$$
\frac{d}{d t}\left|u_{1}(t)-u_{2}(t)\right|^{2} \leq\left((2 m)^{-1}\left(L_{a}(R)\right)^{2}|l|^{2}\left\|u_{2}(t)\right\|^{2}+2 \eta\right)\left|u_{1}(t)-u_{2}(t)\right|^{2} .
$$

Therefore,

$$
\left|u_{1}(t)-u_{2}(t)\right|^{2} \leq \exp \left[\int_{\tau}^{t}\left((2 m)^{-1}\left(L_{a}(R)\right)^{2}|l|^{2}\left\|u_{2}(s)\right\|^{2}+2 \eta\right) d s\right]\left|u_{1 \tau}-u_{2 \tau}\right|^{2},
$$

whence both statements, uniqueness (when $u_{1 \tau}=u_{2 \tau}$ ) and continuity w.r.t. initial data follow. 
Step 2. Existence of weak solution. Assume that $u_{\tau} \in L^{2}(\Omega)$ and $h \in L_{l o c}^{2}\left(\mathbb{R} ; H^{-1}(\Omega)\right)$. By the spectral theory, we can consider $\left\{w_{j}\right\} \subset H_{0}^{1}(\Omega)$, a Hilbert basis of $L^{2}(\Omega)$ formed by eigenfunctions of $-\Delta$ with zero Dirichlet boundary condition in $\Omega$, with corresponding eigenvalues $0<\lambda_{1} \leq \lambda_{2} \leq \ldots$. For every integer $n \geq 1$, we denote by $u_{n}\left(t ; \tau, u_{\tau}\right)=\sum_{j=1}^{n} \varphi_{n j}(t) w_{j}, u_{n}(\cdot)$ for short, the (Galerkin approximation) solution of

$$
\left\{\begin{array}{l}
\frac{d}{d t}\left(u_{n}(t), w_{j}\right)+a\left(l\left(u_{n}(t)\right)\right)\left(\left(u_{n}(t), w_{j}\right)\right)=\left(f\left(u_{n}(t)\right), w_{j}\right)+\left\langle h(t), w_{j}\right\rangle, \quad t>\tau \\
\left(u_{n}(\tau), w_{j}\right)=\left(u_{\tau}, w_{j}\right), \quad j=1, \ldots, n .
\end{array}\right.
$$

Multiplying by $\varphi_{n j}(t)$ in (8), summing from $j=1$ to $n$, and using (2), we obtain

$$
\frac{d}{d t}\left|u_{n}(t)\right|^{2}+2 m\left\|u_{n}(t)\right\|^{2} \leq 2\left(f\left(u_{n}(t)\right), u_{n}(t)\right)+2\left\langle h(t), u_{n}(t)\right\rangle \quad \text { a.e. } t>\tau .
$$

By the Young inequality and (3),

$$
\frac{d}{d t}\left|u_{n}(t)\right|^{2}+m\left\|u_{n}(t)\right\|^{2} \leq \frac{4 C_{f}^{2}|\Omega|}{\lambda_{1} m}+\frac{4 C_{f}^{2}}{\lambda_{1} m}\left|u_{n}(t)\right|^{2}+\frac{2}{m}\|h(t)\|_{*}^{2} \quad \text { a.e. } t>\tau .
$$

Hence, from the Gronwall lemma we deduce that $\left\{u_{n}\right\}$ is bounded in $L^{\infty}\left(\tau, T ; L^{2}(\Omega)\right) \cap L^{2}\left(\tau, T ; H_{0}^{1}(\Omega)\right)$.

As a consequence, $\left\{-a\left(l\left(u_{n}\right)\right) \Delta u_{n}\right\}$ is bounded in $L^{2}\left(\tau, T ; H^{-1}(\Omega)\right)$, and using (3), $\left\{f\left(u_{n}\right)\right\}$ is bounded in $L^{2}\left(\tau, T ; L^{2}(\Omega)\right)$. Then, it is not difficult to prove that $\left\{u_{n}^{\prime}\right\}$ is bounded in $L^{2}\left(\tau, T ; H^{-1}(\Omega)\right)$. Therefore, from compactness arguments and the Aubin-Lions lemma, there exist a subsequence of $\left\{u_{n}\right\}$ (relabelled the same) and $u \in L^{\infty}\left(\tau, T ; L^{2}(\Omega)\right) \cap L^{2}\left(\tau, T ; H_{0}^{1}(\Omega)\right)$ with $u^{\prime} \in L^{2}\left(\tau, T ; H^{-1}(\Omega)\right)$, such that

$$
\begin{aligned}
u_{n} & \stackrel{*}{ } u \quad \text { weakly-star in } L^{\infty}\left(\tau, T ; L^{2}(\Omega)\right), \\
u_{n} & \rightarrow u \quad \text { weakly in } L^{2}\left(\tau, T ; H_{0}^{1}(\Omega)\right), \\
u_{n}^{\prime} & \rightarrow u^{\prime} \quad \text { weakly in } L^{2}\left(\tau, T ; H^{-1}(\Omega)\right), \\
f\left(u_{n}\right) & \rightarrow \xi_{1} \quad \text { weakly in } L^{2}\left(\tau, T ; L^{2}(\Omega)\right), \\
a\left(l\left(u_{n}\right)\right) u_{n} & \rightarrow \xi_{2} \quad \text { weakly in } L^{2}\left(\tau, T ; H_{0}^{1}(\Omega)\right), \\
u_{n} & \rightarrow u \quad \text { strongly in } L^{2}\left(\tau, T ; L^{2}(\Omega)\right), \\
u_{n}(x, t) & \rightarrow u(x, t) \quad \text { a.e. }(x, t) \in \Omega \times(\tau, T), \\
u_{n}(t) & \rightarrow u(t) \quad \text { strongly in } L^{2}(\Omega) \text { a.e. } t \in(\tau, T)
\end{aligned}
$$

for all $T>\tau$.

Then, from [23, Lemme 1.3, p. 12], it is not difficult to prove that $\xi_{1}=f(u)$ and $\xi_{2}=a(l(u)) u$. Thus, we can pass to the limit in (8), and thanks to the fact that $\cup_{n \in \mathbb{N}} \operatorname{span}\left[w_{1}, \ldots, w_{n}\right]$ is dense in $H_{0}^{1}(\Omega)$, we conclude that $u$ is a weak solution.

Step 3. Regularizing effect and strong solution. Integrating in (10) between $\tau$ and $T$, in particular

$$
m \int_{\tau}^{T}\left\|u_{n}(s)\right\|^{2} d s \leq\left|u_{\tau}\right|^{2}+\frac{4 C_{f}^{2}|\Omega|}{\lambda_{1} m}(T-\tau)+\frac{4 C_{f}^{2}}{\lambda_{1} m} \int_{\tau}^{T}\left|u_{n}(s)\right|^{2} d s+\frac{2}{m} \int_{\tau}^{T}\|h(s)\|_{*}^{2} d s .
$$

Assuming now that $h \in L_{l o c}^{2}\left(\mathbb{R} ; L^{2}(\Omega)\right)$, multiplying by $\lambda_{j} \varphi_{n j}(t)$ in (8), summing from $j=1$ to $n$, and using (2), (3), and the Young and Poincaré inequalities, we obtain

$$
\frac{d}{d t}\left\|u_{n}(t)\right\|^{2}+m\left|-\Delta u_{n}(t)\right|^{2} \leq \frac{4 C_{f}^{2}|\Omega|}{m}+\frac{4 C_{f}^{2}}{\lambda_{1} m}\left\|u_{n}(t)\right\|^{2}+\frac{2}{m}|h(t)|^{2} \quad \text { a.e. } t \geq \tau .
$$


Integrating the previous expression between $s$ and $t$, with $\tau \leq s \leq t \leq T$,

$$
\begin{aligned}
& \left\|u_{n}(t)\right\|^{2}+m \int_{s}^{t}\left|-\Delta u_{n}(r)\right|^{2} d r \\
& \quad \leq \frac{4 C_{f}^{2}|\Omega|(T-\tau)}{m}+\frac{4 C_{f}^{2}}{\lambda_{1} m} \int_{s}^{t}\left\|u_{n}(r)\right\|^{2} d r+\frac{2}{m} \int_{s}^{t}|h(r)|^{2} d r+\left\|u_{n}(s)\right\|^{2} .
\end{aligned}
$$

In particular, integrating again with respect to $s$ between $\tau$ and $t$, it holds

$$
(t-\tau)\left\|u_{n}(t)\right\|^{2} \leq \frac{4 C_{f}^{2}|\Omega|(T-\tau)^{2}}{m}+\left(\frac{4 C_{f}^{2}(T-\tau)}{\lambda_{1} m}+1\right) \int_{\tau}^{T}\left\|u_{n}(r)\right\|^{2} d r+\frac{2(T-\tau)}{m} \int_{\tau}^{T}|h(r)|^{2} d r .
$$

Therefore, for all $t \in[\varepsilon+\tau, T]$ with $\varepsilon \in(0, T-\tau)$, it holds

$$
\left\|u_{n}(t)\right\|^{2} \leq \frac{4 C_{f}^{2}|\Omega|(T-\tau)^{2}}{\varepsilon m}+\left(\frac{4 C_{f}^{2}(T-\tau)+\lambda_{1} m}{\varepsilon \lambda_{1} m}\right) \int_{\tau}^{T}\left\|u_{n}(r)\right\|^{2} d r+\frac{2(T-\tau)}{\varepsilon m} \int_{\tau}^{T}|h(r)|^{2} d r .
$$

From this and (11) we deduce that the sequence $\left\{u_{n}\right\}$ is bounded in $L^{\infty}\left(\varepsilon+\tau, T ; H_{0}^{1}(\Omega)\right)$.

On the other hand, taking $s=\varepsilon$ and $t=T$ in (12), and using the previous estimates, we obtain that $\left\{u_{n}\right\}$ is bounded in $L^{2}(\tau+\varepsilon, T ; D(-\Delta))$. As a consequence, $\left\{u_{n}^{\prime}\right\}$ is bounded in $L^{2}(\tau+$ $\left.\varepsilon, T ; L^{2}(\Omega)\right)$, and thanks to the uniqueness of the weak solution, it holds that $\left\{u_{n}\right\}$ converges to $u$ weakly in $L^{2}(\tau+\varepsilon, T ; D(-\Delta))$ and $\left\{u_{n}^{\prime}\right\}$ converges to $u^{\prime}$ weakly in $L^{2}\left(\tau+\varepsilon, T ; L^{2}(\Omega)\right)$. Thus, $u \in L^{2}(\tau+\varepsilon, T ; D(-\Delta)) \cap C\left((\tau, T] ; H_{0}^{1}(\Omega)\right)$.

The case in which the initial datum $u_{\tau}$ belongs to $H_{0}^{1}(\Omega)$ allows to simplify the above estimates in a standard way, and the solution becomes actually strong.

\section{Stationary solutions and their stability}

In this section we are interested in proving that problem (1) admits stationary solutions under some extra assumptions. In order to do this, we will use a corollary of the Brouwer fixed point theorem. Also, under additional suitable requirements, we will prove that in fact the stationary solution is unique and is globally asymptotically exponentially stable.

As we aim now to deal with stationary (steady-state) solutions for the problem, we assume that $h$ is time-independent, i.e. $h \in H^{-1}(\Omega)$. We also assume in this paragraph that the functions $a$ and $f$ are globally Lipschitz, with respective Lipschitz constants $L_{a}, L_{f} \geq 0$.

Once that we consider the evolutionary problem (1) under the above assumptions, by a stationary solution to the problem, we mean an element $u^{*} \in H_{0}^{1}(\Omega)$ such that

$$
a\left(l\left(u^{*}\right)\right)\left(\left(u^{*}, v\right)\right)=\left(f\left(u^{*}\right), v\right)+\langle h, v\rangle \quad \forall v \in H_{0}^{1}(\Omega) .
$$

We then can prove the following result concerning existence, uniqueness, and regularity of stationary solutions to the problem (the idea of the proof is close to that in [25]).

Theorem 6. Assume that the functions $a$ and $f$ are globally Lipschitz functions, with Lipschitz constants $L_{a}$ and $L_{f}$ respectively, (2) is satisfied, $h \in H^{-1}(\Omega), l \in L^{2}(\Omega)$, and $m>\lambda_{1}^{-1} L_{f}$. Then:

1. Problem (13) admits at least one solution. Moreover, any solution $u^{*}$ to (13) satisfies

$$
\left\|u^{*}\right\| \leq \Upsilon:=\frac{\lambda_{1}^{-1 / 2}|\Omega|^{1 / 2}|f(0)|+\|h\|_{*}}{m-\lambda_{1}^{-1} L_{f}}
$$

In addition, if $h \in L^{2}(\Omega)$, then the solutions given above belong in fact to $D(-\Delta)$. 
2. Besides, if we assume

$$
\lambda_{1}^{-1 / 2}|l| L_{a} \Upsilon<m-\lambda_{1}^{-1} L_{f}
$$

problem (13) possesses a unique solution.

Proof. We split the proof of the two statements.

Step 1. Existence. As in the proof of Theorem 5, we choose the Hilbert basis $\left\{w_{j}\right\}$ of $L^{2}(\Omega)$, formed by the eigenvectors (with corresponding eigenvalues $\left\{\lambda_{j}\right\}$ ) of the operator $-\Delta$ in $\Omega$ with zero Dirichlet boundary condition. For each $n \geq 1$, let us denote $V_{n}=\operatorname{span}\left[w_{1}, \ldots, w_{n}\right]$, with the inner product $((\cdot, \cdot))$ and norm $\|\cdot\|$. Then, for all $n \geq 1$, we define the operators $R_{n}: V_{n} \rightarrow V_{n}$ as follows,

$$
\left(\left(R_{n} u, v\right)\right)=\langle-a(l(u)) \Delta u, v\rangle-(f(u), v)-\langle h, v\rangle \quad \forall u, v \in V_{n} .
$$

Since the right hand side is a continuous linear map from $V_{n}$ to $\mathbb{R}$, the Riesz Theorem ensures that each $R_{n} u \in V_{n}$ is well defined. Now, let us check that $R_{n}$ is continuous.

Indeed, observe that

$$
\begin{aligned}
\left(\left(R_{n} u-R_{n} \tilde{u}, v\right)\right) & =\langle-a(l(u)) \Delta u+a(l(\tilde{u})) \Delta \tilde{u}-f(u)+f(\tilde{u}), v\rangle \\
& =\langle-a(l(u)) \Delta(u-\tilde{u})+(a(l(\tilde{u}))-a(l(u))) \Delta \tilde{u}, v\rangle+(f(\tilde{u})-f(u), v) \\
& \leq\left(M\|u-\tilde{u}\|+L_{a}|l| \lambda_{1}^{-1 / 2}\|\tilde{u}-u\|\|\tilde{u}\|+L_{f} \lambda_{1}^{-1}\|\tilde{u}-u\|\right)\|v\|
\end{aligned}
$$

for all $u, \tilde{u}, v \in V_{n}$, thanks to the assumptions on the functions $a, f$, and $l$. Therefore,

$$
\left\|R_{n} u-R_{n} \tilde{u}\right\| \leq\left(M+\lambda_{1}^{-1 / 2}|l| L_{a}\|\tilde{u}\|+\lambda_{1}^{-1} L_{f}\right)\|\tilde{u}-u\| .
$$

for all $u, \tilde{u} \in V_{n}$. This proves that $R_{n}: V_{n} \rightarrow V_{n}$ is continuous.

On the other hand,

$$
\begin{aligned}
\left(\left(R_{n} u, u\right)\right) & =\langle-a(l(u)) \Delta u, u\rangle-(f(u), u) \pm(f(0), u)-\langle h, u\rangle \\
& \geq m\|u\|^{2}-\lambda_{1}^{-1} L_{f}\|u\|^{2}-|f(0)| \lambda_{1}^{-1 / 2}|\Omega|^{1 / 2}\|u\|-\|h\|_{*}\|u\|
\end{aligned}
$$

for all $u \in V_{n}$.

Hence, we obtain $\left(\left(R_{n} u, u\right)\right) \geq 0$ for all $u \in V_{n}$ such that $\|u\|=\Upsilon$, the value given in (14). Consequently, by a corollary of the Brouwer fixed point theorem (see [23, Lemme 4.3, p. 53]), for each $n \geq 1$ there exists $u_{n} \in V_{n}$ such that $R_{n}\left(u_{n}\right)=0$, with $\left\|u_{n}\right\| \leq \Upsilon$.

Therefore, we deduce that

$$
\left\langle-a\left(l\left(u_{n}\right)\right) \Delta u_{n}, v\right\rangle=\left(f\left(u_{n}\right), v\right)+\langle h, v\rangle \quad \forall v \in V_{n} .
$$

Since the sequence $\left\{u_{n}\right\}$ is bounded in $H_{0}^{1}(\Omega)$, the compact embedding of $H_{0}^{1}(\Omega)$ in $L^{2}(\Omega)$ allows us to extract a subsequence $\left\{u_{n^{\prime}}\right\} \subset\left\{u_{n}\right\}$ that converges weakly in $H_{0}^{1}(\Omega)$ and strongly in $L^{2}(\Omega)$ to an element $u^{*} \in H_{0}^{1}(\Omega)$, which also verifies (14). Passing to the limit in the above equality, and taking into account the assumptions on the functions $a, f$, and $l$, we obtain that $u^{*}$ is a solution to (13).

The a priori estimate (14). It is deduced in a similar way as we argued in (16).

Regularity. Assume now that $h \in L^{2}(\Omega)$, and consider any stationary solution $u^{*}$ of the problem (13). We must prove that $u^{*} \in D(-\Delta)$. Obviously, $u^{*}$ is solution to the evolutionary problem (1) with initial datum $u(\tau)=u^{*}$. Therefore, by the regularity result in Theorem $5, u^{*} \in D(-\Delta)$.

Step 2. Uniqueness. Let us suppose that $u_{1}$ and $u_{2}$ are two solutions to (13). Then,

$$
\left\langle-a\left(l\left(u_{1}\right)\right) \Delta u_{1}+a\left(l\left(u_{2}\right)\right) \Delta u_{2}, v\right\rangle=\left(f\left(u_{1}\right)-f\left(u_{2}\right), v\right)
$$

for all $v \in H_{0}^{1}(\Omega)$. Introducing $\pm a\left(l\left(u_{1}\right)\right) \Delta u_{2}$ and taking $v=u_{1}-u_{2}$ as test function, we obtain

$$
m\left\|u_{1}-u_{2}\right\|^{2} \leq\left(\lambda_{1}^{-1 / 2}|l| L_{a}\left\|u_{2}\right\|+\lambda_{1}^{-1} L_{f}\right)\left\|u_{1}-u_{2}\right\|^{2} .
$$


If $u_{1} \neq u_{2}$, we can simplify the above expression, dropping the factor $\left\|u_{1}-u_{2}\right\|^{2}$. But then, using the a priori estimate (14) for $u_{2}$, we would arrive at the opposite inequality to that one in (15), what is a contradiction. Thus, it must be $u_{1}=u_{2}$, and the proof is concluded.

Our second result in this section establishes the global asymptotic exponential stability of the unique stationary solution obtained previously.

Theorem 7. Under the assumptions in Theorem 6, and if (15) holds, then, the following estimate holds for the difference of any solution to (1) and the unique solution $u^{*}$ to (13):

$$
\left|u\left(t ; \tau, u_{\tau}\right)-u^{*}\right|^{2} \leq e^{-\lambda(t-\tau)}\left|u_{\tau}-u^{*}\right|^{2} \quad \forall t \geq \tau
$$

where $\lambda=2 \lambda_{1}\left(m-\lambda_{1}^{-1} L_{f}-\lambda_{1}^{-1 / 2}|l| L_{a} \Upsilon\right)>0$.

Proof. For short, denote by $u(\cdot)$ the weak solution of (1). Then, from the energy equality,

$$
\frac{1}{2} \frac{d}{d t}\left|u(t)-u^{*}\right|^{2}=\left\langle a(l(u(t))) \Delta u(t)-a\left(l\left(u^{*}\right)\right) \Delta u^{*}+f(u(t))-f\left(u^{*}\right), u(t)-u^{*}\right\rangle \quad \text { a.e. } t \in(\tau, T) .
$$

Introducing $\pm a(l(u)) \Delta u^{*}$, and using (2), the Poincaré inequality, and the fact that the functions $a$ and $f$ are globally Lipschitz, it holds

$$
\begin{aligned}
\frac{1}{2} \frac{d}{d t}\left|u(t)-u^{*}\right|^{2} & =-a(l(u(t)))\left\|u(t)-u^{*}\right\|^{2}+\left\langle\left(a(l(u(t)))-a\left(l\left(u^{*}\right)\right)\right) \Delta u^{*}+f(u(t))-f\left(u^{*}\right), u(t)-u^{*}\right\rangle \\
& \leq\left(-m+\lambda_{1}^{-1 / 2}|l| L_{a}\left\|u^{*}\right\|+L_{f} \lambda_{1}^{-1}\right)\left\|u(t)-u^{*}\right\|^{2}
\end{aligned}
$$

From (14), (15), and thanks once again to the Poincaré inequality, we arrive at

$$
\frac{d}{d t}\left|u(t)-u^{*}\right|^{2} \leq-\lambda\left|u(t)-u^{*}\right|^{2} \quad \text { a.e. } t>\tau
$$

where $\lambda$ is given in the statement. The proof is therefore complete.

\section{Abstract results on the theory of Pullback Attractors}

The aim of this section is to recall briefly the main results from the theory of pullback attractors which will be used in the sequel (e.g. cf. $[5,6,19,27])$.

Consider given a metric space $\left(X, d_{X}\right)$, and let us denote $\mathbb{R}_{d}^{2}=\left\{(t, \tau) \in \mathbb{R}^{2}: \tau \leq t\right\}$.

\section{Definition 8.}

(a) A process on $X$ (also called a two-parameter semigroup) is a mapping $\mathbb{R}_{d}^{2} \times X \ni(t, \tau, x) \mapsto$ $U(t, \tau) x \in X$ such that $U(\tau, \tau) x=x$ for any $(\tau, x) \in \mathbb{R} \times X$, and $U(t, s)(U(s, r) x)=U(t, r) x$ for any $r \leq s \leq t$ and all $x \in X$.

(b) A process $U$ on $X$ is said to be

1. continuous if for any pair $(t, \tau) \in \mathbb{R}_{d}^{2}$, the mapping $U(t, \tau): X \rightarrow X$ is continuous;

2. strong-weak (also known as norm-to-weak) continuous if for any pair $(t, \tau) \in \mathbb{R}_{d}^{2}$, the map $U(t, \tau)$ is continuous from $X$ with the strong topology into $X$ with the weak topology;

3. closed if for any pair $(t, \tau) \in \mathbb{R}_{d}^{2}$, and any sequence $\left\{x_{n}\right\} \subset X$, if $x_{n} \rightarrow x \in X$ and $U(t, \tau) x_{n} \rightarrow y \in X$, then $U(t, \tau) x=y$. 
It is clear that every continuous process is strong-weak continuous, and every strong-weak continuous process is closed.

Let us denote by $\mathcal{P}(X)$ the family of all nonempty subsets of $X$ and consider $\mathcal{D}$ a nonempty class of families parameterized in time $\widehat{D}=\{D(t): t \in \mathbb{R}\} \subset \mathcal{P}(X)$ and a family of nonempty sets $\widehat{D}_{0}=\left\{D_{0}(t): t \in \mathbb{R}\right\} \subset \mathcal{P}(X)$. Observe what we do not require any additional condition on these sets such as compactness or boundedness.

Definition 9. A process $U$ on $X$ is said to be pullback $\mathcal{D}$-asymptotically compact if for any $t \in \mathbb{R}$, any $\widehat{D} \in \mathcal{D}$, and any sequences $\left\{\tau_{n}\right\} \subset(-\infty, t]$ and $\left\{x_{n}\right\} \subset X$ satisfying $\tau_{n} \rightarrow-\infty$ and $x_{n} \in D\left(\tau_{n}\right)$ for all $n$, the sequence $\left\{U\left(t, \tau_{n}\right) x_{n}\right\}$ is relatively compact in $X$.

The family $\widehat{D}_{0}=\left\{D_{0}(t): t \in \mathbb{R}\right\} \subset \mathcal{P}(X)$ is pullback $\mathcal{D}$-absorbing for the process $U$ on $X$ if for any $t \in \mathbb{R}$ and any $\widehat{D} \in \mathcal{D}$, there exists $\tau_{0}(\widehat{D}, t)<t$ such that $U(t, \tau) D(\tau) \subset D_{0}(t)$ for all $\tau \leq \tau_{0}(\widehat{D}, t)$.

Observe that in the above definition $\widehat{D}_{0}$ does not necessarily belong to the class $\mathcal{D}$.

Definition 10. The family $\mathcal{A}_{\mathcal{D}}=\left\{\mathcal{A}_{\mathcal{D}}(t): t \in \mathbb{R}\right\} \subset \mathcal{P}(X)$ is said to be the minimal pullback $\mathcal{D}$-attractor for the process $U$ if it satisfies the following properties:

1. for any $t \in \mathbb{R}$, the set $\mathcal{A}_{\mathcal{D}}(t)$ is a nonempty compact subset of $X$;

2. $\mathcal{A}_{\mathcal{D}}$ is pullback $\mathcal{D}$-attracting, i.e. $\lim _{\tau \rightarrow-\infty}$ dist $_{X}\left(U(t, \tau) D(\tau), \mathcal{A}_{\mathcal{D}}(t)\right)=0$ for all $\widehat{D} \in \mathcal{D}, t \in \mathbb{R}$, where dist $_{X}(\cdot, \cdot)$ denotes the Hausdorff semi-distance in $X$ between two subsets of $X$;

3. $\mathcal{A}_{\mathcal{D}}$ is invariant, i.e. $U(t, \tau) \mathcal{A}_{\mathcal{D}}(\tau)=\mathcal{A}_{\mathcal{D}}(t)$ for all $\tau \leq t$

4. $\mathcal{A}_{\mathcal{D}}$ is minimal in the sense that if $\widehat{C}=\{C(t): t \in \mathbb{R}\} \subset \mathcal{P}(X)$ is a family of closed sets which is pullback $\mathcal{D}$-attracting, then $\mathcal{A}_{\mathcal{D}}(t) \subset C(t)$ for all $t \in \mathbb{R}$.

The following result ensures the existence of a minimal pullback attractor (cf. [19, Theorem 3.11]).

Theorem 11. Consider a closed process $U: \mathbb{R}_{d}^{2} \times X \rightarrow X$, a universe $\mathcal{D}$ in $\mathcal{P}(X)$, and a family $\widehat{D}_{0}=\left\{D_{0}(t): t \in \mathbb{R}\right\} \subset \mathcal{P}(X)$ which is pullback $\mathcal{D}$-absorbing for $U$ and assume also that $U$ is pullback $\widehat{D}_{0}$-asymptotically compact.

Then, the family $\mathcal{A}_{\mathcal{D}}=\left\{\mathcal{A}_{\mathcal{D}}(t): t \in \mathbb{R}\right\}$ defined by $\mathcal{A}_{\mathcal{D}}(t)={\overline{\bigcup_{\widehat{D} \in \mathcal{D}} \Lambda(\widehat{D}, t)}}^{X}$ for $t \in \mathbb{R}$, where $\Lambda(\widehat{D}, t)=\bigcap_{s \leq t}{\overline{\bigcup_{\tau \leq s} U(t, \tau) D(\tau)}}^{X}$ and $\overline{\{\ldots\}}^{X}$ denotes the closure in $X$, is the minimal pullback $\mathcal{D}$-attractor for the process $U$. Moreover, if $\widehat{\mathcal{D}}_{0} \in \mathcal{D}$, then $\mathcal{A}_{\mathcal{D}}(t) \subset{\overline{D_{0}(t)}}^{X}$ for all $t \in \mathbb{R}$.

Let us denote by $\mathcal{D}_{F}^{X}$ the universe of fixed nonempty bounded subsets of $X$, i.e. the class of all families $\widehat{D}$ of the form $\widehat{D}=\{D(t)=D: t \in \mathbb{R}\}$ with $D$ a fixed nonempty bounded subset of $X$. In the particular case of the universe $\mathcal{D}_{F}^{X}$, the corresponding minimal pullback $\mathcal{D}_{F}^{X}$-attractor for the process $U$ is the pullback attractor defined by Crauel, Debussche, and Flandoli (see [17]).

Then, we have the following relationships between pullback attractors (see [27]).

Corollary 12. Under the assumptions of Theorem 11 , if $\mathcal{D}_{F}^{X} \subset \mathcal{D}$, it holds that both attractors, $\mathcal{A}_{\mathcal{D}_{F}^{X}}$ and $\mathcal{A}_{\mathcal{D}}$, exist and

$$
\mathcal{A}_{\mathcal{D}_{F}^{X}}(t) \subset \mathcal{A}_{\mathcal{D}}(t) \quad \forall t \in \mathbb{R}
$$

Remark 13. Under the assumptions of the previous corollary, if for some $T \in \mathbb{R}$ the set $\bigcup_{t \leq T} D_{0}(t)$ is a bounded subset of $X$, then

$$
\mathcal{A}_{\mathcal{D}_{F}^{X}}(t)=\mathcal{A}_{\mathcal{D}}(t) \quad \forall t \leq T
$$

The following result allows to compare two attractors for a process under appropriate assumptions (see $[19])$. 
Theorem 14. Consider $\left\{X_{i}, d_{X_{i}}\right\}_{i=1,2}$ two metric spaces such that $X_{1} \subset X_{2}$ with continuous injection, and for $i=1,2$, let $\mathcal{D}_{i}$ be a universe in $\mathcal{P}\left(X_{i}\right)$, with $\mathcal{D}_{1} \subset \mathcal{D}_{2}$. Suppose that there exists a map $U$ that acts as a process in both cases, i.e. $U: \mathbb{R}_{d}^{2} \times X_{i} \rightarrow X_{i}$ for $i=1,2$ is a process.

For each $t \in \mathbb{R}$, let us denote

$$
\mathcal{A}_{i}(t)=\varlimsup_{\widehat{D}_{i} \in \mathcal{D}_{i}} \Lambda_{i}\left(\widehat{D}_{i}, t\right){ }^{X_{i}}, \quad i=1,2,
$$

where the subscript $i$ in the symbol of the omega-limit set $\Lambda_{i}$ is used to denote the dependence on the respective topology.

Then,

$$
\mathcal{A}_{1}(t) \subset \mathcal{A}_{2}(t) \quad \forall t \in \mathbb{R} .
$$

Suppose moreover that the two following conditions are satisfied:

(i) $\mathcal{A}_{1}(t)$ is a compact subset of $X_{1}$ for all $t \in \mathbb{R}$,

(ii) for any $\widehat{D}_{2} \in \mathcal{D}_{2}$ and any $t \in \mathbb{R}$, there exist a family $\widehat{D}_{1} \in \mathcal{D}_{1}$ and a $t_{\widehat{D}_{1}}^{*}$ such that $U$ is pullback $\widehat{D}_{1}$-asymptotically compact, and for any $s \leq t_{\widehat{\mathcal{D}}_{1}}^{*}$ there exists a $\tau_{s}<s$ such that

$$
U(s, \tau) D_{2}(\tau) \subset D_{1}(s) \quad \forall \tau \leq \tau_{s} .
$$

Then, it holds

$$
\mathcal{A}_{1}(t)=\mathcal{A}_{2}(t) \quad \forall t \in \mathbb{R}
$$

\section{$5 \quad$ Pullback attractors in $L^{2}(\Omega)$}

Now, we get rid of the special and somehow strong assumptions imposed in Section 3 for the study of stationary solutions and their global exponential stability. Our aim here is to come back to the initial setting in Section 2, complete it with some more general assumptions, and to study the long-time behaviour of the solutions to $(1)$ in $L^{2}(\Omega)$, according to the results on pullback attractors recalled in Section 4.

Thanks to Theorem 5 , we can define a process $U: \mathbb{R}_{d}^{2} \times L^{2}(\Omega) \rightarrow L^{2}(\Omega)$ as

$$
U(t, \tau) u_{\tau}=u\left(t ; \tau, u_{\tau}\right) \quad \forall u_{\tau} \in L^{2}(\Omega), \quad \forall \tau \leq t,
$$

where $u\left(t ; \tau, u_{\tau}\right)$ is the weak solution to (1).

As a straightforward consequence of Theorem 5, we have that

Proposition 15. Assume that the function a is locally Lipschitz and satisfies (2), $f \in C(\mathbb{R})$ fulfills (3) and (4), and $h \in L_{\text {loc }}^{2}\left(\mathbb{R}, H^{-1}(\Omega)\right)$ and $l \in L^{2}(\Omega)$ are given. Then, the process $U$ is continuous on $L^{2}(\Omega)$.

In what follows, we will make an additional assumption on the function $f$, namely

$$
f(s) s \leq \alpha|s|^{2}+\beta \quad \forall s \in \mathbb{R},
$$

where $\alpha \in\left[0, \lambda_{1} m\right)$ and $\beta \geq 0$. Observe that if the constant $C_{f}$ appearing in the assumption (3) belongs to $\left[0, \lambda_{1} m\right)$, this new assumption would be redundant.

To define a suitable universe in $\mathcal{P}\left(L^{2}(\Omega)\right)$ for our purposes, we first establish the following estimate. 
Lemma 16. Suppose that the function a is locally Lipschitz and satisfies (2), $f \in C(\mathbb{R})$ fulfills (3), (4), and (17), and $h \in L_{\text {loc }}^{2}\left(\mathbb{R} ; H^{-1}(\Omega)\right), l \in L^{2}(\Omega)$, and $u_{\tau} \in L^{2}(\Omega)$ are given. Then, for any $\mu \in\left(0,2\left(\lambda_{1} m-\alpha\right)\right)$, the solution $u$ to (1) satisfies

$$
|u(t)|^{2} \leq \frac{2 \beta|\Omega|}{\mu}+e^{-\mu(t-\tau)}\left|u_{\tau}\right|^{2}+\frac{e^{-\mu t}}{2\left(m-\alpha \lambda_{1}^{-1}\right)-\mu \lambda_{1}^{-1}} \int_{\tau}^{t} e^{\mu s}\|h(s)\|_{*}^{2} d s \quad \forall t \geq \tau .
$$

Proof. From the energy equality, the Cauchy-Schwartz inequality, and by (3) and (17),

$$
\frac{d}{d t}|u(t)|^{2}+\mu|u(t)|^{2}+2 m\|u(t)\|^{2} \leq(2 \alpha+\mu)|u(t)|^{2}+2 \beta|\Omega|+2\|h(t)\|_{*}\|u(t)\| .
$$

Using the Poincaré and Young inequalities,

$$
\frac{d}{d t}|u(t)|^{2}+\mu|u(t)|^{2} \leq 2 \beta|\Omega|+\frac{1}{2 m-(2 \alpha+\mu) \lambda_{1}^{-1}}\|h(t)\|_{*}^{2} .
$$

Multiplying by $e^{\mu t}$ and integrating on $[\tau, t]$, the result follows.

Now, we define a suitable tempered universe in $\mathcal{P}\left(L^{2}(\Omega)\right)$.

Definition 17. For each $\mu>0$, we denote by $\mathcal{D}_{\mu}^{L^{2}}$ the class of all families of nonempty subsets $\widehat{D}=\{D(t): t \in \mathbb{R}\} \subset \mathcal{P}\left(L^{2}(\Omega)\right)$ such that

$$
\lim _{\tau \rightarrow-\infty}\left(e^{\mu \tau} \sup _{v \in D(\tau)}|v|^{2}\right)=0 .
$$

Remark 18. Observe that $\mathcal{D}_{F}^{L^{2}} \subset \mathcal{D}_{\mu}^{L^{2}}$ and $\mathcal{D}_{\mu}^{L^{2}}$ is inclusion-closed, i.e. if $\widehat{D} \in \mathcal{D}_{\mu}^{L^{2}}$ and $\widehat{D}^{\prime}=\left\{D^{\prime}(t)\right.$ : $t \in \mathbb{R}\} \subset \mathcal{P}(X)$ with $D^{\prime}(t) \subset D(t)$ for all $t \in \mathbb{R}$, then $\widehat{D}^{\prime} \in \mathcal{D}_{\mu}^{L^{2}}$.

From the above estimate, if $h$ satisfies a suitable growth condition, it is straightforward to conclude the existence of an absorbing family for an appropriate choice of tempered universe. Namely, we have the following

Proposition 19. Assume that the function a is locally Lipschitz and satisfies (2), $f \in C(\mathbb{R})$ fulfills (3), (4), and (17), $l \in L^{2}(\Omega)$, and $h \in L_{l o c}^{2}\left(\mathbb{R} ; H^{-1}(\Omega)\right)$ satisfies that there exists some $\mu \in\left(0,2\left(\lambda_{1} m-\alpha\right)\right)$ such that

$$
\int_{-\infty}^{0} e^{\mu s}\|h(s)\|_{*}^{2} d s<+\infty .
$$

Then, the family $\widehat{D}_{0}=\left\{D_{0}(t): t \in \mathbb{R}\right\}$ defined by $D_{0}(t)=\bar{B}_{L^{2}}\left(0, R_{L^{2}}^{1 / 2}(t)\right)$, the closed ball in $L^{2}(\Omega)$ of center zero and radius $R_{L^{2}}^{1 / 2}(t)$, where

$$
R_{L^{2}}(t)=1+\frac{2 \beta|\Omega|}{\mu}+\frac{e^{-\mu t}}{2\left(m-\alpha \lambda_{1}^{-1}\right)-\mu \lambda_{1}^{-1}} \int_{-\infty}^{t} e^{\mu s}\|h(s)\|_{*}^{2} d s
$$

is pullback $\mathcal{D}_{\mu}^{L^{2}}$-absorbing for the process $U: \mathbb{R}_{d}^{2} \times L^{2}(\Omega) \rightarrow L^{2}(\Omega)$. Moreover, $\widehat{D}_{0} \in \mathcal{D}_{\mu}^{L^{2}}$.

In order to prove the existence of minimal pullback attractor for the process $U: \mathbb{R}_{d}^{2} \times L^{2}(\Omega) \rightarrow$ $L^{2}(\Omega)$, we need to check that the process $U$ is pullback $\mathcal{D}_{\mu}^{L^{2}}$-asymptotically compact. For that, let us firstly establish some useful estimates. 
Lemma 20. Under the assumptions of Proposition 19, for any $t \in \mathbb{R}$ and $\widehat{D} \in \mathcal{D}_{\mu}^{L^{2}}$, there exists $\tau_{1}(\widehat{D}, t)<t-2$ such that for any $\tau \leq \tau_{1}(\widehat{D}, t)$ and any $u_{\tau} \in D(\tau)$, we obtain

$$
\left\{\begin{aligned}
\left|u\left(r ; \tau, u_{\tau}\right)\right|^{2} & \leq \rho_{1}(t) \quad \forall r \in[t-2, t], \\
\int_{r-1}^{r}\left\|u\left(\theta ; \tau, u_{\tau}\right)\right\|^{2} d \theta & \leq \rho_{2}(t) \quad \forall r \in[t-1, t], \\
\int_{r-1}^{r}\left\|u^{\prime}\left(\theta ; \tau, u_{\tau}\right)\right\|_{*}^{2} d \theta & \leq \rho_{3}(t) \quad \forall r \in[t-1, t],
\end{aligned}\right.
$$

where

$$
\begin{aligned}
& \rho_{1}(t)=1+\frac{2 \beta|\Omega|}{\mu}+\frac{e^{-\mu(t-2)}}{2\left(m-\alpha \lambda_{1}^{-1}\right)-\mu \lambda_{1}^{-1}} \int_{-\infty}^{t} e^{\mu \theta}\|h(\theta)\|_{*}^{2} d \theta, \\
& \rho_{2}(t)=\frac{1}{m-\alpha \lambda_{1}^{-1}}\left(2 \beta|\Omega|+\rho_{1}(t)+\frac{1}{m-\alpha \lambda_{1}^{-1}} \max _{r \in[t-1, t]} \int_{r-1}^{r}\|h(\theta)\|_{*}^{2} d \theta\right), \\
& \rho_{3}(t)=3\left(M^{2} \rho_{2}(t)+2 C_{f}^{2} \lambda_{1}^{-1}\left(|\Omega|+\rho_{1}(t)\right)+\max _{r \in[t-1, t]} \int_{r-1}^{r}\|h(\theta)\|_{*}^{2} d \theta\right) .
\end{aligned}
$$

Proof. The first inequality in (19) as well as the expression of $\rho_{1}$ follow by arguing as in the proof of Lemma 16, if $\tau \leq \tau_{1}(\widehat{D}, t)<t-2$ (far enough pull back in time) due to our choice of tempered universe, taking into account (18). Notice that indeed this estimate also holds for the Galerkin approximations already used in the proof of Theorem 5 .

For the other two inequalities in (19), we will prove them for the Galerkin approximations, and then, passing to the limit, we will obtain the same estimates for the solution.

Recall that from the energy equality for the Galerkin approximation $u_{n}$, at light of (2), we obtained (9). Therefore, by (17) and the Poincaré inequality,

$$
\frac{d}{d t}\left|u_{n}(t)\right|^{2}+2\left(m-\alpha \lambda_{1}^{-1}\right)\left\|u_{n}(t)\right\|^{2} \leq 2 \beta|\Omega|+2\left\langle h(t), u_{n}(t)\right\rangle \quad \text { a.e. } t>\tau .
$$

By the Young inequality,

$$
\frac{d}{d t}\left|u_{n}(t)\right|^{2}+\left(m-\alpha \lambda_{1}^{-1}\right)\left\|u_{n}(t)\right\|^{2} \leq 2 \beta|\Omega|+\frac{1}{m-\alpha \lambda_{1}^{-1}}\|h(t)\|_{*}^{2} \quad \text { a.e. } t>\tau .
$$

Integrating on $[r-1, r]$ for all $r \in[t-1, t]$, if $\tau \leq \tau_{1}(\widehat{D}, t)$ and $u_{\tau} \in D(\tau)$, it yields

$$
\begin{aligned}
\int_{r-1}^{r}\left\|u_{n}(\theta)\right\|^{2} d \theta & \leq \frac{1}{m-\alpha \lambda_{1}^{-1}}\left(\left|u_{n}(r-1)\right|^{2}+2 \beta|\Omega|+\frac{1}{m-\alpha \lambda_{1}^{-1}} \int_{r-1}^{r}\|h(\theta)\|_{*}^{2} d \theta\right) \\
& \leq \rho_{2}(t),
\end{aligned}
$$

where $\rho_{2}(t)$ is the expression given in the statement, thanks to the first inequality in (19) for $u_{n}$.

Taking into account the equation (8) satisfied by $u_{n}$, that the functions $a$ and $f$ satisfy (2) and (3) respectively, that $-\Delta$ is an isometric isomorphism from $H_{0}^{1}(\Omega)$ into $H^{-1}(\Omega)$, and the already proved first two estimates of (19) for $u_{n}$, we deduce that, for all $r \in[t-1, t], \tau \leq \tau_{1}(\widehat{D}, t), u_{\tau} \in D(\tau)$, and for any $n \geq 1$,

$$
\begin{aligned}
\int_{r-1}^{r}\left\|u_{n}^{\prime}(\theta)\right\|_{*}^{2} d \theta & \leq 3 \int_{r-1}^{r} a\left(l\left(u_{n}(\theta)\right)\right)^{2}\left\|u_{n}(\theta)\right\|^{2} d \theta+\frac{3}{\lambda_{1}} \int_{r-1}^{r}\left|f\left(u_{n}(\theta)\right)\right|^{2} d \theta+3 \int_{r-1}^{r}\|h(\theta)\|_{*}^{2} d \theta \\
& \leq \rho_{3}(t)
\end{aligned}
$$


where $\rho_{3}(t)$ is the expression given in the statement.

Using the facts (proved in Theorem 5$)$ that $\left\{u_{n}\right\}$ converges to $u\left(\cdot ; \tau, u_{\tau}\right)$ weakly in $L^{2}\left(r-1, r ; H_{0}^{1}(\Omega)\right.$ ) for all $r \in[t-1, t]$, and $\left\{u_{n}^{\prime}\right\}$ converges to $u^{\prime}\left(\cdot ; \tau, u_{\tau}\right)$ weakly in $L^{2}\left(r-1, r ; H^{-1}(\Omega)\right)$ for all $r \in[t-1, t]$, from (20) and (21), and passing to the limit, we conclude the result.

Now we apply an energy method with continuous functions (e.g. cf. [19, 28, 26, 21]) in order to obtain the pullback asymptotic compactness in $L^{2}(\Omega)$ for the universe $\mathcal{D}_{\mu}^{L^{2}}$.

Lemma 21. Under the assumptions of Proposition 19, the process $U: \mathbb{R}_{d}^{2} \times L^{2}(\Omega) \rightarrow L^{2}(\Omega)$ is pullback $\mathcal{D}_{\mu}^{L^{2}}$-asymptotically compact.

Proof. Let us fix $t \in \mathbb{R}$, a family $\widehat{D} \in \mathcal{D}_{\mu}^{L^{2}}$, a sequence $\left\{\tau_{n}\right\} \subset(-\infty, t-2]$ with $\tau_{n} \rightarrow-\infty$ and $u_{\tau_{n}} \in D\left(\tau_{n}\right)$ for all $n$. We will prove that the sequence $\left\{u\left(t ; \tau_{n}, u_{\tau_{n}}\right)\right\}$ is relatively compact in $L^{2}(\Omega)$. For short, we will denote $u^{n}(\cdot)=u\left(\cdot ; \tau_{n}, u_{\tau_{n}}\right)$.

Thanks to Lemma 20, (2), and (3), we know that there exists $\tau_{1}(\widehat{D}, t)<t-2$ satisfying that, if $n_{1} \geq 1$ is such that $\tau_{n} \leq \tau_{1}(\widehat{D}, t)$ for all $n \geq n_{1},\left\{u^{n}\right\}_{n \geq n_{1}}$ is bounded in $L^{\infty}\left(t-2, t ; L^{2}(\Omega)\right) \cap$ $L^{2}\left(t-2, t ; H_{0}^{1}(\Omega)\right),\left\{-a\left(l\left(u^{n}\right)\right) \Delta u^{n}\right\}_{n \geq n_{1}}$ is bounded in $L^{2}\left(t-2, t ; H^{-1}(\Omega)\right),\left\{f\left(u^{n}\right)\right\}_{n \geq n_{1}}$ is bounded in $L^{2}\left(t-2, t ; L^{2}(\Omega)\right)$, and $\left\{\left(u^{n}\right)^{\prime}\right\}_{n \geq n_{1}}$ is bounded in $L^{2}\left(t-2, t ; H^{-1}(\Omega)\right)$. Then, using the Aubin-Lions lemma, there exists $u \in L^{\infty}\left(t-2, t ; L^{2}(\Omega)\right) \cap L^{2}\left(t-2, t ; H_{0}^{1}(\Omega)\right)$ with $u^{\prime} \in L^{2}\left(t-2, t ; H^{-1}(\Omega)\right)$, such that for a subsequence (relabelled the same) it holds

$$
\left\{\begin{array}{rll}
u^{n} & * & \\
u^{n} & \rightarrow u & \text { weakly-star in } L^{\infty}\left(t-2, t ; L^{2}(\Omega)\right), \\
\left(u^{n}\right)^{\prime} & \rightarrow u^{\prime} & \text { weakly in } L^{2}\left(t-2, t ; H_{0}^{1}(\Omega)\right), \\
u^{n} & \rightarrow u & \text { weakly in } L^{2}\left(t-2, t ; H^{-1}(\Omega)\right), \\
u^{n}(s) & \rightarrow u(s) & \text { strongly in } L^{2}\left(t-2, t ; L^{2}(\Omega)\right), \\
f\left(u^{n}\right) & \rightarrow f(u) & \text { weakly in } L^{2}\left(t-2, t ; L^{2}(\Omega)\right), \\
a\left(l\left(u^{n}\right)\right) u^{n} & \rightarrow a(l(u)) u & \text { weakly in } L^{2}\left(t-2, t ; H_{0}^{1}(\Omega)\right) .
\end{array}\right.
$$

Observe that $u \in C\left([t-2, t] ; L^{2}(\Omega)\right)$, and due to (22) it is not difficult to prove that $u$ satisfies (5) in the interval $(t-2, t)$.

Since $\left\{\left(u^{n}\right)^{\prime}\right\}_{n \geq n_{1}}$ is bounded in $L^{2}\left(t-2, t ; H^{-1}(\Omega)\right)$, we have that $\left\{u^{n}\right\}_{n \geq n_{1}}$ is equicontinuous in $H^{-1}(\Omega)$, on $[t-2, t]$. In addition, as $\left\{u^{n}\right\}_{n \geq n_{1}}$ is bounded in $C\left([t-2, t] ; L^{2}(\Omega)\right)$ and the embedding $L^{2}(\Omega) \subset H^{-1}(\Omega)$ is compact, by the Ascoli-Arzelà Theorem, we obtain (for another subsequence, relabelled again the same)

$$
u^{n} \rightarrow u \quad \text { strongly in } C\left([t-2, t] ; H^{-1}(\Omega)\right) .
$$

Using that $\left\{u^{n}\right\}_{n \geq n_{1}}$ is bounded in $C\left([t-2, t] ; L^{2}(\Omega)\right)$, we have that for any sequence $\left\{s_{n}\right\} \subset[t-2, t]$ with $s_{n} \rightarrow s_{*}$, it holds

$$
u^{n}\left(s_{n}\right) \rightarrow u\left(s_{*}\right) \quad \text { weakly in } L^{2}(\Omega),
$$

where we have used (23) to identify the weak limit.

If we prove that

$$
u^{n} \rightarrow u \quad \text { strongly in } C\left([t-1, t] ; L^{2}(\Omega)\right),
$$

in particular we will have that $\left\{u\left(t ; \tau_{n}, u_{\tau_{n}}\right)\right\}$ is relatively compact in $L^{2}(\Omega)$.

We establish (25) by contradiction. We suppose that there exist $\varepsilon>0$, a sequence $\left\{t_{n}\right\} \subset[t-1, t]$, without loss of generality converging to some $t_{*}$, with

$$
\left|u^{n}\left(t_{n}\right)-u\left(t_{*}\right)\right| \geq \varepsilon \quad \forall n \geq 1 .
$$

From (24) we deduce

$$
\left|u\left(t_{*}\right)\right| \leq \liminf _{n \rightarrow+\infty}\left|u^{n}\left(t_{n}\right)\right|
$$


On the other hand, using the energy equality (6), the Young inequality, (2) and (17), the estimate

$$
|z(s)|^{2} \leq|z(r)|^{2}+2 \beta|\Omega|(s-r)+\frac{1}{2\left(m-\alpha \lambda_{1}^{-1}\right)} \int_{r}^{s}\|h(\theta)\|_{*}^{2} d \theta \quad \forall t-2 \leq r \leq s \leq t
$$

holds with $z$ replaced by $u$ or any $u^{n}$.

Now we define the functions

$$
\begin{aligned}
J_{n}(s) & =\left|u^{n}(s)\right|^{2}-2 \beta|\Omega| s-\frac{1}{2\left(m-\alpha \lambda_{1}^{-1}\right)} \int_{t-2}^{s}\|h(r)\|_{*}^{2} d r, \\
J(s) & =|u(s)|^{2}-2 \beta|\Omega| s-\frac{1}{2\left(m-\alpha \lambda_{1}^{-1}\right)} \int_{t-2}^{s}\|h(r)\|_{*}^{2} d r .
\end{aligned}
$$

It holds from the regularity of $u$ and all $u^{n}$ and the above inequality that these functions $J$ and $J_{n}$ are continuous and non-increasing on $[t-2, t]$.

Observe now that using (22), it holds

$$
J_{n}(s) \rightarrow J(s) \text { a.e. } s \in(t-2, t) .
$$

Hence, there exists a sequence $\left\{\tilde{t}_{k}\right\} \subset\left(t-2, t_{*}\right)$ such that $\tilde{t}_{k} \rightarrow t_{*}$ when $k \rightarrow+\infty$ and

$$
\lim _{n \rightarrow+\infty} J_{n}\left(\tilde{t}_{k}\right)=J\left(\tilde{t}_{k}\right) \quad \forall k \geq 1 .
$$

Fix an arbitrary value $\epsilon>0$. From the continuity of $J$ on $[t-2, t]$, there exists $k(\epsilon) \geq 1$ such that

$$
\left|J\left(\tilde{t}_{k}\right)-J\left(t_{*}\right)\right| \leq \epsilon / 2 \quad \forall k \geq k(\epsilon) .
$$

Now consider $n(\epsilon) \geq 1$ such that

$$
t_{n} \geq \tilde{t}_{k(\epsilon)} \quad \text { and } \quad\left|J_{n}\left(\tilde{t}_{k(\epsilon)}\right)-J\left(\tilde{t}_{k(\epsilon)}\right)\right| \leq \epsilon / 2 \quad \forall n \geq n(\epsilon) .
$$

Then, since all $J_{n}$ are non-increasing, we deduce

$$
\begin{aligned}
J_{n}\left(t_{n}\right)-J\left(t_{*}\right) & \leq J_{n}\left(\tilde{t}_{k(\epsilon)}\right)-J\left(t_{*}\right) \\
& \leq\left|J_{n}\left(\tilde{t}_{k(\epsilon)}\right)-J\left(t_{*}\right)\right| \\
& \leq\left|J_{n}\left(\tilde{t}_{k(\epsilon)}\right)-J\left(\tilde{t}_{k(\epsilon)}\right)\right|+\left|J\left(\tilde{t}_{k(\epsilon)}\right)-J\left(t_{*}\right)\right| \\
& \leq \epsilon \quad \forall n \geq n(\epsilon) .
\end{aligned}
$$

As $\epsilon>0$ is arbitrary, from above we deduce $\lim \sup _{n \rightarrow+\infty} J_{n}\left(t_{n}\right) \leq J\left(t_{*}\right)$, and thus,

$$
\limsup _{n \rightarrow+\infty}\left|u^{n}\left(t_{n}\right)\right| \leq\left|u\left(t_{*}\right)\right| \text {. }
$$

From this, (24), and (27), it holds that $\left\{u^{n}\left(t_{n}\right)\right\}$ converges to $u\left(t_{*}\right)$ strongly in $L^{2}(\Omega)$, in contradiction with (26). Therefore, (25) is proved.

As a consequence of the previous results, we obtain the following theorem, which is the main result of this section.

Theorem 22. Suppose that the function a is locally Lipschitz and satisfies (2), $f \in C(\mathbb{R})$ fulfills (3), (4), and (17), $l \in L^{2}(\Omega)$, and $h \in L_{\text {loc }}^{2}\left(\mathbb{R} ; H^{-1}(\Omega)\right)$ satisfies condition (18) for some $\mu \in\left(0,2\left(\lambda_{1} m-\right.\right.$ $\alpha)$ ). Then, there exist the minimal pullback $\mathcal{D}_{F}^{L^{2}}$-attractor $\mathcal{A}_{\mathcal{D}_{F}^{L^{2}}}=\left\{\mathcal{A}_{\mathcal{D}_{F}^{L^{2}}}(t): t \in \mathbb{R}\right\}$, and the minimal 
pullback $\mathcal{D}_{\mu}^{L^{2}}$-attractor $\mathcal{A}_{\mathcal{D}_{\mu}^{L^{2}}}=\left\{\mathcal{A}_{\mathcal{D}_{\mu}^{L^{2}}}(t): t \in \mathbb{R}\right\}$, for the process $U: \mathbb{R}_{d}^{2} \times L^{2}(\Omega) \rightarrow L^{2}(\Omega)$. The family $\mathcal{A}_{\mathcal{D}_{\mu}^{L^{2}}}$ belongs to $\mathcal{D}_{\mu}^{L^{2}}$, and it holds

$$
\mathcal{A}_{\mathcal{D}_{F}^{L^{2}}}(t) \subset \mathcal{A}_{\mathcal{D}_{\mu}^{L^{2}}}(t) \subset \bar{B}_{L^{2}}\left(0, R_{L^{2}}^{1 / 2}(t)\right) \quad \forall t \in \mathbb{R}
$$

Moreover, if $h$ satisfies

$$
\sup _{s \leq 0}\left(e^{-\mu s} \int_{-\infty}^{s} e^{\mu \theta}\|h(\theta)\|_{*}^{2} d \theta\right)<+\infty
$$

then both attractors coincide, i.e. $\mathcal{A}_{\mathcal{D}_{F}^{L^{2}}}(t)=\mathcal{A}_{\mathcal{D}_{\mu}^{L^{2}}}(t)$ for all $t \in \mathbb{R}$.

Proof. The existence of $\mathcal{A}_{\mathcal{D}_{\mu}^{L^{2}}}, \mathcal{A}_{\mathcal{D}_{F}^{L^{2}}}$ and the first relation between both attractors is a consequence of the abstract results in the previous section, namely, by Corollary 12. Indeed, the continuity of the process (cf. Proposition 15), the relation between the universes (cf. Remark 18), and two main ingredients, absorbing family (cf. Proposition 19) and asymptotic compactness (cf. Lemma 21) hold.

The relation between the family $\mathcal{A}_{\mathcal{D}_{\mu}^{L^{2}}}$ and $\widehat{D}_{0}$ is a direct consequence of Theorem 11. The family $\mathcal{A}_{\mathcal{D}_{\mu}^{L^{2}}}$ belongs to $\mathcal{D}_{\mu}^{L^{2}}$ since $\widehat{D}_{0} \in \mathcal{D}_{\mu}^{L^{2}}$, the set $D_{0}(t)$ is closed for all $t \in \mathbb{R}$, and the universe $\mathcal{D}_{\mu}^{L^{2}}$ is inclusion-closed.

Finally, the coincidence of both families of attractors under the assumption (28) follows from Remark 13, since the expression of $R_{L^{2}}(t)$ given in Proposition 19 satisfies that for each $T \in \mathbb{R}$, $\cup_{t \leq T} R_{L^{2}}(t)$ is bounded.

\section{Remark 23.}

(i) Observe that condition (28) is equivalent to $\sup _{s \leq 0} \int_{s-1}^{s}\|h(\theta)\|_{*}^{2} d \theta<+\infty$.

(ii) Notice that if $h \in L_{\text {loc }}^{2}\left(\mathbb{R} ; H^{-1}(\Omega)\right)$ satisfies condition (18) for some $\mu \in\left(0,2\left(\lambda_{1} m-\alpha\right)\right)$, then it also satisfies

$$
\int_{-\infty}^{0} e^{\sigma s}\|h(s)\|_{*}^{2} d s<+\infty \quad \forall \sigma \in\left(\mu, 2\left(\lambda_{1} m-\alpha\right)\right) .
$$

Thus, under the assumptions of Theorem 22, for any $\sigma \in\left(\mu, 2\left(\lambda_{1} m-\alpha\right)\right)$ there exists the corresponding minimal pullback $\mathcal{D}_{\sigma}^{L^{2}}$-attractor, $\mathcal{A}_{\mathcal{D}_{\sigma}^{L^{2}}}$. By Theorem 14 , since $\mathcal{D}_{\mu}^{L^{2}} \subset \mathcal{D}_{\sigma}^{L^{2}}$, it is evident that, for any $t \in \mathbb{R}, \mathcal{A}_{\mathcal{D}_{\mu}^{L^{2}}}(t) \subset \mathcal{A}_{\mathcal{D}_{\sigma}^{L^{2}}}(t)$ for all $\sigma \in\left(\mu, 2\left(\lambda_{1} m-\alpha\right)\right)$. Moreover, if $h$ satisfies (28), then, from the above result and the equivalence pointed out in (i), we have $\mathcal{A}_{\mathcal{D}_{F}^{L 2}}(t)=\mathcal{A}_{\mathcal{D}_{\mu}^{L}}(t)=\mathcal{A}_{\mathcal{D}_{\sigma}^{L^{2}}}(t)$ for all $t \in \mathbb{R}$ and any $\sigma \in\left(\mu, 2\left(\lambda_{1} m-\alpha\right)\right)$.

\section{Pullback attractors in $H_{0}^{1}(\Omega)$}

The goal of this section is to improve the results of the previous one, by establishing attraction in $H_{0}^{1}(\Omega)$, and relating new pullback attractors with those proved in Theorem 22 .

To do this, firstly observe that, thanks to Theorem 5 , the restriction of $U$ to $\mathbb{R}_{d}^{2} \times H_{0}^{1}(\Omega)$ defines a process into $H_{0}^{1}(\Omega)$. Since no confusion arises, we will not modify the notation, and continue denoting this process as $U$.

Actually, this process defined on $H_{0}^{1}(\Omega)$ as phase-space still fulfills properties to apply the results of Section 4. The following result shows that the process $U$ is strong-weak continuous in $H_{0}^{1}(\Omega)$.

Proposition 24. Assume that the function a is locally Lipschitz and satisfies (2), $f \in C(\mathbb{R})$ satisfies (3) and (4), $l \in L^{2}(\Omega)$ and $h \in L_{l o c}^{2}\left(\mathbb{R}, L^{2}(\Omega)\right)$. Then, the process $U$ is strong-weak continuous in $H_{0}^{1}(\Omega)$. 
Proof. Consider fixed $(\tau, t) \in \mathbb{R}_{d}^{2}$. Let $\left\{u_{\tau_{n}}\right\}$ be a sequence that converges to $u_{\tau}$ strongly in $H_{0}^{1}(\Omega)$.

On the one hand, by Proposition 15, it holds that the map $U(t, \tau)$ is continuous from $L^{2}(\Omega)$ into $L^{2}(\Omega)$. Therefore,

$$
U(t, \tau) u_{\tau_{n}} \rightarrow U(t, \tau) u_{\tau} \text { strongly in } L^{2}(\Omega) .
$$

On the other hand, using (7), (2), (3), and the Hölder and Young inequalities, it holds

$$
\left\|U(t, \tau) u_{\tau_{n}}\right\|^{2} \leq\left\|u_{\tau_{n}}\right\|^{2}+\frac{2 C_{f}^{2}}{m}\left(\int_{\tau}^{t}\left(|\Omega|+\left|U(s, \tau) u_{\tau_{n}}\right|^{2}\right) d s\right)+\frac{1}{m} \int_{\tau}^{t}|h(s)|^{2} d s .
$$

From (6), and the Gronwall lemma, in particular, a uniform estimate for $\left\{U(\cdot, \tau) u_{\tau_{n}}\right\}$ in $L^{2}\left(\tau, t ; L^{2}(\Omega)\right)$ follows easily. Hence, the sequence $\left\{U(t, \tau) u_{\tau_{n}}\right\}$ is bounded in $H_{0}^{1}(\Omega)$. Then, by the uniqueness of the limit, it holds

$$
U(t, \tau) u_{\tau_{n}} \rightarrow U(t, \tau) u_{\tau} \quad \text { weakly in } H_{0}^{1}(\Omega)
$$

Next result, which is analogous to Lemma 20, establishes some uniform estimates of the solutions in more regular norms in a finite-time interval up to time $t$ when the initial datum is shifted pullback far enough. This will be useful to prove the pullback asymptotic compactness in $H_{0}^{1}(\Omega)$. In order to simplify the statement, let us firstly introduce the following two quantities:

$$
\begin{aligned}
& \rho_{1}^{e x t}(t)=1+\frac{2 \beta|\Omega|}{\mu}+\frac{e^{-\mu(t-3)}}{2\left(m-\alpha \lambda_{1}^{-1}\right)-\mu \lambda_{1}^{-1}} \int_{-\infty}^{t} e^{\mu \theta}\|h(\theta)\|_{*}^{2} d \theta, \\
& \rho_{2}^{e x t}(t)=\frac{1}{m-\alpha \lambda_{1}^{-1}}\left(2 \beta|\Omega|+\rho_{1}^{e x t}(t)+\frac{1}{m-\alpha \lambda_{1}^{-1}} \max _{r \in[t-2, t]} \int_{r-1}^{r}\|h(\theta)\|_{*}^{2} d \theta\right) .
\end{aligned}
$$

[The upper script ext means that these expressions are estimates, close to those in Lemma 20 involving $\rho_{1}$ and $\rho_{2}$, but in an extended interval, as will be indicated in the proof below.]

Lemma 25. Assume that the function a is locally Lipschitz and satisfies (2), $l \in L^{2}(\Omega), f \in C(\mathbb{R})$ satisfies (3), (4), and (17), and $h \in L_{l o c}^{2}\left(\mathbb{R} ; L^{2}(\Omega)\right)$ satisfies (18) for some $\mu \in\left(0,2\left(\lambda_{1} m-\alpha\right)\right)$. Then, for any $t \in \mathbb{R}$ and $\widehat{D} \in \mathcal{D}_{\mu}^{L^{2}}$, there exists $\tau_{2}(\widehat{D}, t)<t-3$, such that for any $\tau \leq \tau_{2}(\widehat{D}, t)$ and any $u_{\tau} \in D(\tau)$, it holds

$$
\left\{\begin{aligned}
\left\|u\left(r ; \tau, u_{\tau}\right)\right\|^{2} & \leq \widetilde{\rho}_{1}(t) \quad \forall r \in[t-2, t], \\
\int_{r-1}^{r}\left|-\Delta u\left(\theta ; \tau, u_{\tau}\right)\right|^{2} d \theta & \leq \widetilde{\rho}_{2}(t) \quad \forall r \in[t-1, t], \\
\int_{r-1}^{r}\left|u^{\prime}\left(\theta ; \tau, u_{\tau}\right)\right|^{2} d \theta & \leq \widetilde{\rho}_{3}(t) \quad \forall r \in[t-1, t],
\end{aligned}\right.
$$

where, taking into account $\left\{\rho_{i}^{e x t}\right\}_{i=1,2}$ from (29), the terms $\left\{\widetilde{\rho}_{i}\right\}_{i=1,2,3}$ are given by

$$
\begin{aligned}
& \tilde{\rho}_{1}(t)=\frac{4 C_{f}^{2}|\Omega|}{m}+\left(1+\frac{4 C_{f}^{2}}{\lambda_{1} m}\right) \rho_{2}^{e x t}(t)+\frac{2}{m} \max _{r \in[t-2, t]} \int_{r-1}^{r}|h(\theta)|^{2} d \theta, \\
& \tilde{\rho}_{2}(t)=\frac{4 C_{f}^{2}|\Omega|}{m^{2}}+\frac{1}{m} \widetilde{\rho}_{1}(t)+\frac{4 C_{f}^{2}}{\lambda_{1} m^{2}} \rho_{2}^{e x t}(t)+\frac{2}{m^{2}} \max _{r \in[t-1, t]} \int_{r-1}^{r}|h(\theta)|^{2} d \theta, \\
& \widetilde{\rho}_{3}(t)=3 M^{2} \widetilde{\rho}_{2}(t)+6 C_{f}^{2}|\Omega|+\frac{6 C_{f}^{2}}{\lambda_{1}} \rho_{2}^{e x t}(t)+3 \max _{r \in[t-1, t]} \int_{r-1}^{r}|h(\theta)|^{2} d \theta .
\end{aligned}
$$


Proof. Let us firstly observe that, analogously as we argued in Lemma 20, we may obtain uniform estimates for solutions in a longer time-interval (useful for our purposes). Namely, there exists $\tau_{2}(\widehat{D}, t)<t-3$, such that for any $\tau \leq \tau_{2}(\widehat{D}, t)$ and any $u_{\tau} \in D(\tau)$, it holds

$$
\begin{aligned}
\left|u\left(r ; \tau, u_{\tau}\right)\right|^{2} & \leq \rho_{1}^{e x t}(t) \quad \forall r \in[t-3, t], \\
\int_{r-1}^{r}\left\|u\left(\theta ; \tau, u_{\tau}\right)\right\|^{2} d \theta & \leq \rho_{2}^{e x t}(t) \quad \forall r \in[t-2, t],
\end{aligned}
$$

where $\left\{\rho_{i}^{\text {ext }}\right\}_{i=1,2}$ are given in (29). Actually, these estimates also hold for the Galerkin approximations $u_{n}\left(\cdot ; \tau, u_{\tau}\right)$.

Taking this into account, we will prove the lemma by obtaining the inequalities for the Galerkin approximations and then passing to the limit.

Now, multiplying by $\lambda_{j} \varphi_{n j}(\theta)$ in (8), summing from $j=1$ to $n$ and using (2), (3), and the Young inequality, we obtain

$$
\frac{d}{d \theta}\left\|u_{n}(\theta)\right\|^{2}+m\left|-\Delta u_{n}(\theta)\right|^{2} \leq \frac{4 C_{f}^{2}|\Omega|}{m}+\frac{4 C_{f}^{2}}{\lambda_{1} m}\left\|u_{n}(\theta)\right\|^{2}+\frac{2}{m}|h(\theta)|^{2} \quad \text { a.e. } \theta>\tau
$$

Integrating between $r$ and $s$ with $\tau \leq r-1 \leq s \leq r$, we obtain in particular

$$
\left\|u_{n}(r)\right\|^{2} \leq\left\|u_{n}(s)\right\|^{2}+\frac{4 C_{f}^{2}|\Omega|}{m}+\frac{4 C_{f}^{2}}{\lambda_{1} m} \int_{r-1}^{r}\left\|u_{n}(\theta)\right\|^{2} d \theta+\frac{2}{m} \int_{r-1}^{r}|h(\theta)|^{2} d \theta .
$$

Integrating the last inequality w.r.t. $s$ on $[r-1, r]$, it holds

$$
\left\|u_{n}(r)\right\|^{2} \leq\left(1+\frac{4 C_{f}^{2}}{\lambda_{1} m}\right) \int_{r-1}^{r}\left\|u_{n}(s)\right\|^{2} d s+\frac{4 C_{f}^{2}|\Omega|}{m}+\frac{2}{m} \int_{r-1}^{r}|h(\theta)|^{2} d \theta,
$$

for all $\tau \leq r-1$.

Therefore, from the estimate on the solutions by $\rho_{2}^{e x t}$ given above, one deduces that

$$
\left\|u_{n}\left(r ; \tau, u_{\tau}\right)\right\|^{2} \leq \widetilde{\rho}_{1}(t) \quad \forall r \in[t-2, t], \quad \tau \leq \tau_{2}(\widehat{D}, t), \quad u_{\tau} \in D(\tau),
$$

where $\widetilde{\rho}_{1}(t)$ is given in the statement.

Now, integrating between $r-1$ and $r$ in (31), we obtain in particular

$$
\int_{r-1}^{r}\left|-\Delta u_{n}(\theta)\right|^{2} d \theta \leq \frac{1}{m}\left\|u_{n}(r-1)\right\|^{2}+\frac{4 C_{f}^{2}|\Omega|}{m^{2}}+\frac{4 C_{f}^{2}}{\lambda_{1} m^{2}} \int_{r-1}^{r}\left\|u_{n}(\theta)\right\|^{2} d \theta+\frac{2}{m^{2}} \int_{r-1}^{r}|h(\theta)|^{2} d \theta
$$

for all $\tau \leq r-1$.

Therefore, for any $n \geq 1$,

$$
\int_{r-1}^{r}\left|-\Delta u_{n}(\theta)\right|^{2} d \theta \leq \widetilde{\rho}_{2}(t) \quad \forall r \in[t-1, t], \quad \tau \leq \tau_{2}(\widehat{D}, t), \quad u_{\tau} \in D(\tau),
$$

where $\widetilde{\rho}_{2}(t)$ is given in the statement.

On the other hand,

$$
\int_{r-1}^{r}\left|u_{n}^{\prime}(\theta)\right|^{2} d \theta \leq 3 \int_{r-1}^{r} a\left(l\left(u_{n}(\theta)\right)\right)^{2}\left|-\Delta u_{n}(\theta)\right|^{2} d \theta+3 \int_{r-1}^{r}\left|f\left(u_{n}(\theta)\right)\right|^{2} d \theta+3 \int_{r-1}^{r}|h(\theta)|^{2} d \theta,
$$

for all $\tau \leq r-1$. Therefore, from (2), (3), and the above estimates, for any $n \geq 1$, it yields

$$
\int_{r-1}^{r}\left|u_{n}^{\prime}(\theta)\right|^{2} d \theta \leq \widetilde{\rho}_{3}(t) \quad \forall r \in[t-1, t], \quad \tau \leq \tau_{2}(\widehat{D}, t), \quad u_{\tau} \in D(\tau)
$$


where $\widetilde{\rho}_{3}(t)$ is given in the statement.

Using that $u \in C\left([t-2, t] ; H_{0}^{1}(\Omega)\right)$ and the facts that $\left\{u_{n}\right\}$ converges to $u\left(\cdot ; \tau, u_{\tau}\right)$ weakly-star in $L^{\infty}\left(t-2, t ; H_{0}^{1}(\Omega)\right)$ and weakly in $L^{2}(r-1, r ; D(-\Delta))$ for all $r \in[t-1, t]$, and $\left\{u_{n}^{\prime}\right\}$ converges to $u^{\prime}\left(\cdot ; \tau, u_{\tau}\right)$ weakly in $L^{2}\left(r-1, r ; L^{2}(\Omega)\right)$ for all $r \in[t-1, t]$, from the above estimates, passing to the limit we conclude (30).

Now, we introduce additional universes, that involve more regularity.

Definition 26. For each $\mu>0$, we will denote by $\mathcal{D}_{\mu}^{L^{2}, H_{0}^{1}}$ the class of all families of nonempty subsets $\widehat{D}_{H_{0}^{1}}=\left\{D(t) \cap H_{0}^{1}(\Omega): t \in \mathbb{R}\right\}$, where $\widehat{D}=\{D(t): t \in \mathbb{R}\} \in \mathcal{D}_{\mu}^{L^{2}}$.

Remark 27. Observe that $\mathcal{D}_{F}^{H_{0}^{1}} \subset \mathcal{D}_{\mu}^{L^{2}, H_{0}^{1}}$ and $\mathcal{D}_{\mu}^{L^{2}, H_{0}^{1}}$ is inclusion-closed.

As a direct consequence of the regularizing effect of the equation when $h \in L_{l o c}^{2}\left(\mathbb{R} ; L^{2}(\Omega)\right.$ ) (cf. Theorem 5) and the existence of a family pullback $\mathcal{D}_{\mu}^{L^{2}}$-absorbing (cf. Proposition 19), the existence of an absorbing family in this universe $\mathcal{D}_{\mu}^{L^{2}, H_{0}^{1}}$ also holds.

Proposition 28. Under the assumptions of Lemma 25, the family

$$
\widehat{D}_{0, H_{0}^{1}}=\left\{\bar{B}\left(0, R_{L^{2}}^{1 / 2}(t)\right) \cap H_{0}^{1}(\Omega): t \in \mathbb{R}\right\}
$$

belongs to $\mathcal{D}_{\mu}^{L^{2}, H_{0}^{1}}$ and for any $t \in \mathbb{R}$ and any $\widehat{D} \in \mathcal{D}_{\mu}^{L^{2}}$, there exists $\tau_{3}(\widehat{D}, t)<t$ such that

$$
U(t, \tau) D(\tau) \subset D_{0, H_{0}^{1}}(t) \quad \forall \tau \leq \tau_{3}(\widehat{D}, t) .
$$

In particular, the family $\widehat{D}_{0, H_{0}^{1}}$ is pullback $\mathcal{D}_{\mu}^{L^{2}, H_{0}^{1}}$-absorbing for the process $U: \mathbb{R}_{d}^{2} \times H_{0}^{1}(\Omega) \rightarrow H_{0}^{1}(\Omega)$.

Now, we apply an energy method analogous to the one we used in the previous section. This will lead to pullback asymptotic compactness of $U$ in $H_{0}^{1}(\Omega)$ for the universe $\mathcal{D}_{\mu}^{L^{2}, H_{0}^{1}}$.

Proposition 29. Under the assumptions of Lemma 25, the process $U: \mathbb{R}_{d}^{2} \times H_{0}^{1}(\Omega) \rightarrow H_{0}^{1}(\Omega)$ is pullback $\mathcal{D}_{\mu}^{L^{2}, H_{0}^{1}}$-asymptotically compact.

Proof. The proof is similar to that of Lemma 21, and we omit it for the sake of brevity. Let us just indicate the main key in this more regular setting: in this case we consider the energy equality (7) and the continuous and non-increasing functions

$$
\begin{aligned}
J_{n}(s) & =\left\|u^{n}(s)\right\|^{2}-\frac{4 C_{f}^{2}|\Omega|}{m} s-\frac{4 C_{f}^{2}}{m} \int_{t-2}^{s}\left|u^{n}(r)\right|^{2} d r-\frac{2}{m} \int_{t-2}^{s}|h(r)|^{2} d r, \\
J(s) & =\|u(s)\|^{2}-\frac{4 C_{f}^{2}|\Omega|}{m} s-\frac{4 C_{f}^{2}}{m} \int_{t-2}^{s}|u(r)|^{2} d r-\frac{2}{m} \int_{t-2}^{s}|h(r)|^{2} d r .
\end{aligned}
$$

As a consequence of the above results, we obtain the existence of minimal pullback attractors for the process $U: \mathbb{R}_{d}^{2} \times H_{0}^{1}(\Omega) \rightarrow H_{0}^{1}(\Omega)$.

Theorem 30. Suppose that the function a is locally Lipschitz and satisfies (2), $f \in C(\mathbb{R})$ fulfills (3), (4), and (17), $l \in L^{2}(\Omega)$, and $h \in L_{\text {loc }}^{2}\left(\mathbb{R}, L^{2}(\Omega)\right)$ verifies (18) for some $\mu \in\left(0,2\left(\lambda_{1} m-\alpha\right)\right)$. Then, there exist the minimal pullback $\mathcal{D}_{F}^{H_{0}^{1}}$-attractor $\mathcal{A}_{\mathcal{D}_{F}^{H_{0}^{1}}}=\left\{\mathcal{A}_{\mathcal{D}_{F}^{H_{0}^{1}}}(t): t \in \mathbb{R}\right\}$ and the minimal pullback 
$\mathcal{D}_{\mu}^{L^{2}, H_{0}^{1}}$-attractor $\mathcal{A}_{\mathcal{D}_{\mu}^{L^{2}, H_{0}^{1}}}=\left\{\mathcal{A}_{\mathcal{D}_{\mu}^{L^{2}, H_{0}^{1}}}(t): t \in \mathbb{R}\right\}$, for the process $U: \mathbb{R}_{d}^{2} \times H_{0}^{1}(\Omega) \rightarrow H_{0}^{1}(\Omega)$, and it holds

$$
\mathcal{A}_{\mathcal{D}_{F}^{H_{0}^{1}}}(t) \subset \mathcal{A}_{\mathcal{D}_{F}^{L^{2}}}(t) \subset \mathcal{A}_{\mathcal{D}_{\mu}^{L^{2}}}(t)=\mathcal{A}_{\mathcal{D}_{\mu}^{L^{2}, H_{0}^{1}}}(t) \quad \forall t \in \mathbb{R},
$$

where $\mathcal{A}_{\mathcal{D}_{F}^{L^{2}}}$ and $\mathcal{A}_{\mathcal{D}_{F}^{L^{2}}}$ are respectively the minimal pullback $\mathcal{D}_{F}^{L^{2}}$-attractor and the minimal pullback $\mathcal{D}_{\mu}^{L^{2}}$-attractor for the process $U: \mathbb{R}_{d}^{2} \times L^{2}(\Omega) \rightarrow L^{2}(\Omega)$, whose existence is guaranteed by Theorem 22 . In particular, it holds the following pullback attraction result

$$
\lim _{\tau \rightarrow-\infty} \operatorname{dist}_{H_{0}^{1}}\left(U(t, \tau) D(\tau), \mathcal{A}_{\mathcal{D}_{\mu}^{L^{2}}}(t)\right)=0 \quad \forall t \in \mathbb{R} \quad \forall \widehat{D} \in \mathcal{D}_{\mu}^{L^{2}} .
$$

Finally, if moreover $h$ satisfies not only (28) but

$$
\sup _{s \leq 0}\left(e^{-\mu s} \int_{-\infty}^{s} e^{\mu r}|h(r)|^{2} d r\right)<+\infty,
$$

then all attractors in (32) coincide, i.e.

$$
\mathcal{A}_{\mathcal{D}_{F}^{H_{0}^{1}}(t)}=\mathcal{A}_{\mathcal{D}_{F}^{L^{2}}}(t)=\mathcal{A}_{\mathcal{D}_{\mu}^{L^{2}}}(t)=\mathcal{A}_{\mathcal{D}_{\mu}^{L^{2}, H_{0}^{1}}}(t) \quad \forall t \in \mathbb{R},
$$

and again, a result of pullback attraction holds, in this case for any bounded subset $B$ of $L^{2}(\Omega)$ :

$$
\lim _{\tau \rightarrow-\infty} \operatorname{dist}_{H_{0}^{1}}\left(U(t, \tau) B, \mathcal{A}_{\mathcal{D}_{F}^{L}}(t)\right)=0 \quad \forall t \in \mathbb{R} .
$$

Proof. The existence of $\mathcal{A}_{\mathcal{D}_{F}^{H_{0}^{1}}}$ and $\mathcal{A}_{\mathcal{D}_{\mu}^{L^{2}, H_{0}^{1}}}$ is a consequence of Corollary 12, since $U$ is strong-weak continuous in $H_{0}^{1}(\Omega)$ (cf. Proposition 24), the relation between the universes (cf. Remark 27), and we have the existence of an absorbing family in $\mathcal{D}_{\mu}^{L^{2}}, H_{0}^{1}$ (cf. Proposition 28) and asymptotic compactness of this universe in the $H^{1}$ norm (cf. Proposition 29).

The chain of inclusions (32) follows from Corollary 12 and Theorem 14. Actually, the equality statement is due to the second part of Theorem 14, by using Proposition 28. Then, (33) is straightforward.

If moreover $h$ satisfies (28), we already proved in Theorem 22 the equality $\mathcal{A}_{\mathcal{D}_{F}^{L^{2}}}(t)=\mathcal{A}_{\mathcal{D}_{\mu}^{L^{2}}}(t)$ for all $t \in \mathbb{R}$. Now, in order to obtain (35), we assume (34), which is a requirement stronger than (28). Therefore, the equality $\mathcal{A}_{\mathcal{D}_{F}^{H_{0}^{1}}}(t)=\mathcal{A}_{\mathcal{D}_{F}^{L^{2}}}(t)$ is again a consequence of Theorem 14 . Indeed, the solutions are coming into a bounded subset of $H_{0}^{1}(\Omega)$ due to the first estimate in Lemma 25 by $\widetilde{\rho}_{1}(t)$ [recall that, analogously as in Remark 23 (i), here (34) is equivalent to $\sup _{s \leq 0} \int_{s-1}^{s}|h(r)|^{2} d r<+\infty$ ]. Then, (36) obviously holds.

\section{Remark 31.}

(i) As a complement to Remark 23, under the assumptions of Theorem 30, we can improve the above result, involving new universes and attractors. Namely, for any $\sigma \in\left(\mu, 2\left(\lambda_{1} m-\alpha\right)\right)$ there exists the corresponding minimal pullback $\mathcal{D}_{\sigma}^{L^{2}, H_{0}^{1}}$-attractor, $\mathcal{A}_{\mathcal{D}_{\sigma}^{L^{2}, H_{0}^{1}}}$, and it holds that $\mathcal{A}_{\mathcal{D}_{\sigma}^{L^{2}}}(t)=\mathcal{A}_{\mathcal{D}_{\sigma}^{L^{2}, H_{0}^{1}}}(t)$ for all $t \in \mathbb{R}$. Moreover, if $h$ satisfies (34), then, after (35), $\mathcal{A}_{\mathcal{D}_{\sigma}^{L^{2}, H_{0}^{1}}}$ coincides with the family $\mathcal{A}_{\mathcal{D}_{F}^{H_{0}^{1}}}$ for any $\sigma \in\left(\mu, 2\left(\lambda_{1} m-\alpha\right)\right)$.

(ii) Under additional conditions, we may restrict ourselves to study the problem in the positive cone of $L^{2}(\Omega)$, that is, $\mathcal{C}_{+}\left(L^{2}(\Omega)\right)=\left\{g \in L^{2}(\Omega): g \geq 0 \quad\right.$ a.e. $\left.\Omega\right\}$, and redefining suitably new classes of (tempered and non-tempered) families. Namely, assuming that $h$ is a positive function and $f \in C\left(\mathbb{R} ; \mathbb{R}_{+}\right)$, a Maximum Principle holds, and $U$ is well-defined from into $\mathcal{C}_{+}\left(L^{2}(\Omega)\right)$, which is important if one is dealing with a biological model. Then, all the results from Sections 5 and 6 can be obtained again analogously, by rearranging the assumptions within this setting. 


\section{Acknowledgments}

While concluding this work, we received the very sad news that Professor Silvano B. de Menezes passed away during a stay at Universidad de Sevilla, visiting our department. We feel extremely sorrow for this news, for him, his family and friends, and the big community of mathematicians that knew him.

The authors would like to thank the referees for a careful reading and comments on a previous version of the manuscript.

This work has been partially supported by Ministerio de Economía y Competitividad (Spain), grant MTM2011-22411, Junta de Andalucía grant P12-FQM-1492. M.H.-C. is a fellow of Programa de FPI del Ministerio de Economía y Competitividad (SPAIN).

\section{References}

[1] A. Andami Ovono, Asymptotic behaviour for a diffusion equation governed by nonlocal interactions, Electron. J. Differential Equations 134 (2010), 01-16.

[2] A. Andami Ovono and A. Rougirel, Elliptic equations with diffusion parameterized by the range of nonlocal interactions, Ann. Mat. Pura Appl. (4) 189 (2010), 163-183.

[3] M. Anguiano, P. E. Kloeden, and T. Lorenz, Asymptotic behaviour of nonlocal reaction-diffusion equations, Nonlinear Anal. 73 (2010), 3044-3057.

[4] J. Billingham, Dynamics of a strongly nonlocal reaction-diffusion population model, Nonlinearity 17 (2004), 313-346.

[5] T. Caraballo, G. Eukaszewicz, and J. Real, Pullback attractors for asymptotically compact nonautonomous dynamical systems, Nonlinear Anal. 64 (2006), 484-498.

[6] T. Caraballo, G. Łukaszewicz, and J. Real, Pullback attractors for non-autonomous 2D-NavierStokes equations in some unbounded domains, C. R. Math. Acad. Sci. Paris 342 (2006), 263-268.

[7] N. H. Chang and M. Chipot, Nonlinear nonlocal evolution problems, RACSAM. Rev. R. Acad. Cienc. Exactas Fís. Nat. Ser. A Mat. 97 (2003), 423-445.

[8] N. H. Chang and M. Chipot, On some model diffusion problems with a nonlocal lower order term, Chinese Ann. Math. Ser. B 24 (2003), 147-166.

[9] M. Chipot and B. Lovat, Some remarks on nonlocal elliptic and parabolic problems, Nonlinear Anal. 30 (1997), 4619-4627.

[10] M. Chipot and B. Lovat, On the asymptotic behaviour of some nonlocal problems, Positivity 3 (1999), 65-81.

[11] M. Chipot and B. Lovat, Existence and uniqueness results for a class of nonlocal elliptic and parabolic problems, Dyn. Contin. Discrete Impuls. Syst. Ser. A Math. Anal. 8 (2001), 35-51.

[12] M. Chipot and L. Molinet, Asymptotic behaviour of some nonlocal diffusion problems, Appl. Anal. 80 (2001), 273-315.

[13] M. Chipot and M. Siegwart, On the asymptotic behaviour of some nonlocal mixed boundary value problems, in Nonlinear Analysis and applications: to V. Lakshmikantam on his 80th birthday, pp. 431-449, Kluwer Acad. Publ., Dordrecht, 2003.

[14] M. Chipot, V. Valente, and G. V. Caffarelli, Remarks on a nonlocal problem involving the Dirichlet energy, Rend. Sem. Mat. Univ. Padova 110 (2003), 199-220. 
[15] M. Chipot and S. Zheng, Asymptotic behavior of solutions to nonlinear parabolic equations with nonlocal terms, Asymptot. Anal. 45 (2005), 301-312.

[16] I. Chueshov, Monotone Random Systems Theory and Applications, Lecture Notes in Mathematics 1779, Springer-Verlag, Berlin, 2002.

[17] H. Crauel, A. Debussche, and F. Flandoli, Random attractors, J. Dynam. Differential Equations 9 (1997), 307-341.

[18] P. Freitas, Nonlocal reaction-diffusion equations, Differential equations with applications to biology (Halifax, NS, 1997), 187-204, Fields Inst. Commun., 21, Amer. Math. Soc., Providence, RI, 1999.

[19] J. García-Luengo, P. Marín-Rubio, and J. Real, Pullback attractors in $V$ for non-autonomous 2D-Navier-Stokes equations and their tempered behaviour, J. Differential Equations 252 (2012), $4333-4356$.

[20] J. García-Melián and J. D. Rossi, A logistic equation with refuge and nonlocal diffusion, Commun. Pure Appl. Anal. 8 (2009), 2037-2053.

[21] A.V. Kapustyan, V.S. Melnik, and J. Valero, Attractors of multivalued dynamical processes generated by phase-field equations, Internat. J. Bifur. Chaos Appl. Sci. Engrg. 13 (2003), 1969-1983.

[22] P.E. Kloeden, Pullback attractors of nonautonomous semidynamical systems, Stoch. Dyn. 3 (2003), 101-112.

[23] J. L. Lions, Quelques Méthodes de Résolution des Problèmes aux Limites Non Lineaires, Dunod, Paris, 1969.

[24] B. Lovat, Etudes de quelques problèmes paraboliques non locaux, Thèse, Université de Metz, 1995.

[25] P. Marín-Rubio, A. M. Marquez-Durán, and J. Real, Three dimensional system of globally modified Navier-Stokes equations with infinite delays, Discrete Contin. Dyn. Syst. Ser. B 14 (2010), 655-673.

[26] P. Marín-Rubio, G. Planas, and J. Real, Asymptotic behaviour of a phase-field model with three coupled equations without uniqueness, J. Differential Equations 246 (2009), 4632-4652.

[27] P. Marín-Rubio and J. Real, On the relation between two different concepts of pullback attractors for non-autonomous dynamical systems, Nonlinear Anal. 71 (2009), 3956-3963.

[28] P. Marín-Rubio and J. Real, Pullback attractors for 2D-Navier-Stokes equations with delays in continuous and sub-linear operators, Discrete Contin. Dyn. Syst. 26 (2010), 989-1006.

[29] S. B. de Menezes, Remarks on weak solutions for a nonlocal parabolic problem, Int. J. Math. Math. Sci. 2006 (2006), 01-10.

[30] Z. Szymańska, C. Morales-Rodrigo, M. Lachowicz, and M. A. J. Chaplain, Mathematical modelling of cancer invasion of tissue: the role and effect of nonlocal interaction, Math. Models Methods Appl. Sci. 19 (2009), 257-281. 